\title{
POLYTOPES, GRAPHS, AND COMPLEXES ${ }^{1,2}$
}

\author{
BY BRANKO GRÜNBAUM
}

Communicated May 15, 1970

During the last few years the theory of convex polytopes has been developing at an ever accelerating pace. As a consequence, many parts of the book "Convex Polytopes" ([CP] = Grünbaum [1967a]), which was essentially completed early in 1966, are by now completely out of date. The same fate befell the surveys by Klee [1966] and Grünbaum [1967b]; even the more recent survey by GrünbaumShephard [1969] has been overtaken in many directions by new results. The new achievements are scattered in the literature, and many of them are still in the preprint stage. Hence it seemed worthwhile to prepare an up-to-date summary of the new results, organized in a form that makes it readable without previous specialized knowledge of the theory of polytopes.

The following pages constitute such a survey of a part of the theory; considerations of time and space precluded coverage of all ramifications of the subject of convex polytopes, but most major directions are included. The first part of the survey deals with problems and questions related to graphs, and underscores the extent to which the investigations of polytopes and graphs influence each other. The second part deals with relations among the numbers of

${ }^{1}$ Expanded and updated version of an address delivered before the Honolulu meeting of the Society by invitation of the Committee to Select Hour Speakers for Western Sectional Meetings, April 9, 1966.

AMS 1970 subject classifications. Primary 52A25, 52A40; Secondary 05C10, 05C $30,05 \mathrm{C} 35,05 \mathrm{C} 99,28 \mathrm{~A} 75,41 \mathrm{~A} 63,50 \mathrm{~B} 15,50 \mathrm{~B} 25,50 \mathrm{D} 25,52 \mathrm{~A} 20,53 \mathrm{C} 65,55 \mathrm{~A} 15$, 55A20, 55B99, 57A35, 57A99, 90С 99, 94A10, 94A20.

Key words and phrases. Polytope, graph, simplicial complex, cell complex, subdivision, path, imbedding, planar graph, polytopal graph, connectedness, Steinitz's characterization of 3-polytopes, simple 3-polytopes, Eberhard's theorem on 3-polytopes, valence, Hamiltonian circuit, Euler characteristic, upper-bound conjecture, cyclic polytope, neighbourly polytopes, face, number of faces, lower-bound conjecture, cubical polytope, central symmetry, triangulated sphere, combinatorial invariants, angle sum, angle deficiency, Grassmann manifold, invariant measure, facet-forming polytope, Steiner point, mean width, mixed volume, constant width, Gale transform, Gale diagram, arrangement of hyperplanes, zonotope, positive dependence, primitive polytope, fixing set, enumeration of polytopes, projectively unique polytope, linearly stable polytope, pseudolines, irrational polytope.

2 Research supported in part by the Office of Naval Research through contract N00014-67-A-0103-0003, and by the National Science Foundation through the Science Development Program Grant GU-2648. 
faces of various dimensions of $d$-polytopes, and with other numbers and measures that may be associated with polytopes. The third part surveys the technique of the so-called Gale diagrams, the results obtainable by it, and related material.

Another consideration that greatly influenced the writing of the present survey was my wish to do something, futile as it may turn out to be, against the spreading blight of tunnel vision and sectarianism. While it is true that most of us must resign ourselves to attempts at research in very sharply specialized fields, it has become acceptable and even fashionable to limit one's total knowledge and interests to the same narrow area-a development I consider disastrous. This trend is being abetted by the increasing use of "key words", "subject classifications" and similar means which-besides their useful aspects - tend to fix even more tightly the specialization blinkers we have become accustomed to wear. Even most of the survey papers published in this Bulletin, though presumably originating in lectures intended for a wide mathematical audience, are written in a style which makes it obvious that their aim is to increase the knowledge of the specialist rather than to enlarge the horizon of the non-specialist.

I believe that the theory of convex polytopes is one of the relatively few actively developing areas which are easily understandable and accessible to non-specialists while simultaneously presenting aspects of interest and stimulating interfaces to a wide variety of mathematical disciplines. I also believe that the future of mathematics does not lie exclusively in the erection of ever-higher towers of abstraction on a few basic truths and ideas. Hence, besides trying to make this survey readable, I have attempted to convey some of the excitement that is to be found in the exploration of a highly-structured field which has as yet not been streamlined into lofty generalities.

To facilitate the use of the survey each of the references is followed by the numbers of the pages of the present text in which that paper or book was quoted. This easily performed bibliographic service to the reader, which is very useful though seldom encountered, goes back at least to Coolidge [1940] and probably to much earlier times. Each reference is also provided with the volume and number or page of Mathematical Reviews in which it was reviewed.

The author would greatly appreciate any comments, remarks, or corrections. Particularly welcome would be indications of omissions, which are almost unavoidable in surveys of areas as widely scattered as that of convex polytopes. 
A few words concerning the terminology. The setting of the survey is, almost exclusively, the $n$-dimensional Euclidean space $E^{n}$. A poly tope is the convex hull of a finite set of points of $E^{n}$. If a polytope has dimension $d$ we call it a $d$-polytope. Intersections of a polytope $P$ with supporting hyperplanes are polytopes, called faces of $P$; faces of dimension $k$ are called $k$-faces. For a $d$-polytope $P$, where $d \geqq 2$, the $(d-1)$-faces are called facets of $P$, the 1 -faces are known as edges, and the 0 -faces as vertices. $P$ itself and the empty set $\varnothing$ are considered to be "improper faces" of $P$. A $d$-polytope with $d+1$ vertices is called a $d$-simplex. A $d$-polytope is simplicial if all its proper faces are simplices; $P$ is simple if each vertex belongs to precisely $d$ facets.

1. Polytopal complexes, Steinitz's theorem, and the combinatorial theory of 3-polytopes. The present section is devoted mainly to 3 -polytopes. However, since some of the results and problems generalize in a meaningful way to higher dimensions, we shall start by giving the appropriate definitions, and the known theorems, in the general case.

1.1. Polytopal complexes. A topological polytope $P$ is a homeomorphic image of a convex polytope $P^{\prime}$, endowed with a facial structure carried over to $P$ by the homeomorphism from the facial structure of $P^{\prime}$. (For example, a solid ball in $E^{3}$, with a triangulation of its boundary defined by the equator and $k$ meridians, is a topological 3-polytope $P$ for which $P^{\prime}$ is the $k$-sided bipyramid.)

A geometric [topological] cell complex $\mathrm{e}=\left\{C_{i} \mid i \in I\right\}$ is a finite family of polytopes [topological polytopes] in some Euclidean space $E^{n}$ such that

(i) Every face of a member of $\mathfrak{e}$ is itself a member of $\mathfrak{C}$;

(ii) The intersection of any two members of $\mathbb{C}$ is a face of each of them.

The number of $k$-faces (i.e., $k$-dimensional members) of a (geometric or topological) complex $\mathbb{e}$ shall be denoted by $f_{k}(\mathbb{C})$. If $f_{k}(\mathfrak{C}) \neq 0$ but $f_{i}(\mathfrak{e})=0$ for all $i>k$, we call $\mathfrak{e}$ a $k$-complex. If each face of $\mathfrak{e}$ is a simplex, $\mathcal{C}$ is called simplicial.

Two complexes $\mathfrak{e}$ and $\mathfrak{e}^{\prime}$ are called isomorphic [dual] to each other provided there exists a one-to-one correspondence between their non-empty members which is inclusion preserving [inclusion reversing].

A 1-complex is called a graph; it is geometric if and only if all its edges (that is, 1-faces) are straight-line segments. (Note that in this terminology a graph has no loops and no "multiple edges".)

A $k$-complex $\mathrm{C}$ is geometrically [topologically] imbeddable in $E^{n}$ pro- 
vided there exists in $E^{n}$ a geometric [topological] cell-complex isomorphic to e. A graph imbeddable in $E^{2}$ is called planar.

If $P$ is a $d$-polytope and if $k$ satisfies $0 \leqq k \leqq d$, the set of all faces of $P$ of dimension at most $k$ is a geometric cell $k$-complex; it is called the $k$-skeleton of $P$ and denoted $\operatorname{skel}_{k} P$. The 1 -skeleton of $P$ is also called the graph of $P$; the $(d-1)$-skeleton of $P$ is called the boundary complex of $P$ and denoted by $B(P)$.

A $k$-complex $\mathcal{C}$ is $d$-polytopal provided it is isomorphic to $\operatorname{skel}_{k} P$ for some $d$-polytope $P$.

An interesting but unsolved and probably very hard problem is the characterization of $d$-poly topal $k$-complexes. At present, a number of necessary conditions (that is, nontrivial properties of the skeleta of $d$-polytopes) are known, but only for $d \leqq 3$ has a complete characterization been obtained (see Subsection 1.2).

We begin with a survey of the known properties of skeleta of $d$-polytopes. We recall that a graph $G$ is said to be $k$-connected provided it has at least 2 vertices, and any pair of vertices of $G$ are the endpoints of $k$ paths, disjoint except for the endpoints.

Theorem 1.1. (Balinski [1961]; for a weaker result see RemezŠteinberg [1967].) The graph of a d-polytope is d-connected.

Various extensions of Theorem 1.1 are known. Sallee [1967] obtained results on the degree of connectedness of the higher-dimensional skeleta of $d$-polytopes. Related investigations may be found in Hering [1969]. Larman-Mani [1970a] considered disjoint paths between a number of prescribed pairs of vertices; their results partially extend previous work of Watkins [1968], Mesner-Watkins [1966] and Watkins-Mesner [1967] on similar questions. One of the results of Larman-Mani [1970a] is

Theorem 1.2. If $m=\left[\frac{1}{3}(d+1)\right]$ pairs of distinct vertices $\left(a_{1}, b_{1}\right), \cdots$, $\left(a_{m}, b_{m}\right)$ of a d-polytope $P$ are given, there exist in the graph of $P m$ disjoint paths such that the ith path connects $a_{i}$ to $b_{i}$.

Larman-Mani [1970a] conjecture that even [d/2] pairs could be prescribed; easy examples show that this number may not be increased in general; however, for simplicial $d$-polytopes they prove that Theorem 1.2 remains valid even if $m=\left[\frac{1}{2}(d+1)\right]$.

A different connectivity property of graphs of $d$-polytopes was investigated by Klee [1964a].

Another direction of investigation of $d$-polytopal graphs started with the result: 
THEOREM 1.3. (Grünbaum-Motzkin [1963a].) The graph of each $d$ polytope contains as subgraph a subdivision of the complete graph with $d+1$ vertices.

Strengthenings of 1.3 were obtained by Barnette [1967]; related results also by Larman-Mani [1970a]. Similar ideas in the theory of abstract graphs were explored by Halin [1966], Mader [1967], [1968], and Jung [1967], [1970b]; for more recent problems and results related to Theorems 1.2 and 1.3 see Grünbaum [1970a], Mani [1970a], Jung [1970a].

A generalization of 1.3 to higher-dimensional skeleta of $d$-polytopes (which also yields a simple proof of Theorem 1.3) is given in Grünbaum [1965].

An additional series of results and problems concerning polytopal complexes centers on imbeddability in spaces of various dimensions. We recall (Flores [1932], [1933], van Kampen [1932]) that there exist simplicial $n$-complexes not (topologically) imbeddable in $E^{2 n}$, though each simplicial $n$-complex is (quite trivially) geometrically imbeddable in $E^{2 n+1}$. (See Zaks [1968], [1969] and Grünbaum [1969d] for some related results.)

An extension of this is the following result of M. A. Perles (see [CP, p. 204]):

THEOREM 1.4. If $\mathfrak{e}$ is a geometric cell k-complex in some Euclidean space, then $\mathcal{C}$ is geometrically imbeddable in $E^{2 k+1}$.

It should be noted that it is easy to construct examples of topological cell complexes (of any dimension $\geqq 2$ ) which are not geometrically imbeddable in any Euclidean space. Moreover, it is possible that a cell complex is geometrically imbeddable in $E^{d}$ and topologically imbeddable in $E^{n}$, with $n<d$, without being geometrically imbeddable in $E^{n}$ (see [CP, $\$ \$ 11.1$ and 11.5$]$ where additional results and references to the older literature may be found, and Grünbaum-Sreedharan [1967]). For skeleta of polytopes the situation is much simpler. Let $a(\mathfrak{C})[b(\mathbb{C})]$ denote the minimal dimension of a Euclidean space in which the complex $\mathcal{C}$ is topologically [geometrically] imbeddable. For $1 \leqq k \leqq d$ we define

$$
\begin{aligned}
a(k, d) & =d & & \text { if } \quad k \leqq d \leqq k+1, \\
& =d-1 & & \text { if } \quad k+2 \leqq d \leqq 2 k+2, \\
& =2 k+1 & & \text { if } 2 k+2 \leqq d .
\end{aligned}
$$

Then, extending the results of van Kampen [1932] and Chrislock [1966] concerning simplices, we have [CP, p. 204]: 
TheOREM 1.5. If $P$ is a d-polytope then

$$
a\left(\operatorname{skel}_{k} P\right)=b\left(\operatorname{skel}_{k} P\right)=a(k, d) .
$$

Though the skeleta of $d$-polytopes have not been characterized for $d \geqq 4$, we have the following uniqueness results [CP, $\$ \$ 12.2$ and 12.3]:

Theorem 1.6. If $k \geqq\left[\frac{1}{2} d\right]$, if $P$ and $P^{\prime}$ are polytopes, $\operatorname{dim} P=d$, and if skel $_{k} P$ is isomorphic to skel $_{k} P^{\prime}$, then $\operatorname{dim} P^{\prime}=d$.

Theorem 1.7. If $P$ and $P^{\prime}$ are polytopes, $\operatorname{dim} P=d$, and if skel $_{d-2} P$ is isomorphic to skel s-2 $_{2} P^{\prime}$, then $P$ and $P^{\prime}$ are isomorphic.

The last result may be interpreted as a generalization of the theorem of Whitney [1932] concerning the uniqueness of imbeddings of 3-connected planar graphs in the plane.

If $k<\left[\frac{1}{2} d\right]$, the result of Theorem 1.6 is no longer valid. For the different possible types of "ambiguity" in the polytopal realizations of skeleta of polytopes, and for some special cases of "unambiguity", see Grünbaum-Motzkin [1963a], Klee [1964a], Grünbaum [1963a], [CP, Chapters 11 and 12], and Barnette [1970a].

Of chief interest for the sequel of the present exposition is the special case $d=3$. The above results imply, in particular, that the graph of each 3-polytope is planar and 3-connected. As we shall see next, those conditions are also sufficient for a graph to be 3-polytopal.

1.2. Steinitz's theorem. The graphs of convex polygons (i.e. 2-polytopes) are trivially characterized as simple circuits. The only nontrivial case in which a characterization of $d$-polytopal graphs is known concerns $d=3$. This characterization is given in the following theorem, which is essentially due to Steinitz [1922]:

TheOREM 1.8. $A$ graph $G$ is 3-polytopal if and only if $G$ is planar and 3-connected.

The proof of Theorem 1.8 establishes, moreover, the isomorphism of any two 3-polytopes with isomorphic graphs. ${ }^{3}$ It shows also that if $G$ is any 3-polytopal graph imbedded in the plane, and if $P$ is a 3 -polytope with graph isomorphic to $G$, then the boundary complex $B(P)$ is isomorphic to the 2-complex determined in the plane by $G$. Hence the "countries" determined by $G$ correspond-in their incidence relations and their numbers of edges - to the 2-faces of $P$.

The significance of Steinitz's theorem stems from the fact that if a 3-connected graph $G$ may be drawn (imbedded) in the plane, then it corresponds to a 3-polytope, the combinatorial structure of which

3 See Note 1 on page 1183. 
is completely determined by $G$. Therefore many questions concerning 3-polytopes may be investigated by considering planar graphs. The importance of this reduction possibility will be amply demonstrated in the sequel.

Two aspects of Steinitz's theorem deserve special mention. First, it is a rather deep result, the proof of which is far from trivial (see below). Second, it has been involved in much wishful thinking and quite a few gross errors. Indeed, it is rather obvious that Theorem 1.8 (or some result similar to it) is needed if one wishes to deduce results concerning 3-polytopes from results on planar graphs. Nevertheless, many authors either offered fallaciously naive arguments instead of proofs (see the critique on pp. 56-57 of Steinitz [1922]), or else ignored the necessity of proving anything (see, for example, Schlegel [1893], Schoute [1905, pp. 22, 29]). In an analogous problem concerning 4-polytopes, Brückner [1909] also ignores the problem which is in that case much deeper and still unsolved. No analogue of Steinitz's theorem is known for 4-polytopes, even if the attention is restricted to simplicial ones (see [CP, \$11.5], Grünbaum-Sreedharan [1967] and Barnette-Wegner [1970]). The disregard for the distinction between geometric and topological cell complexes leads Brückner [1909] into an error in the enumeration of simple 4-polytopes with 8 facets (see Grünbaum-Sreedharan [1967], Barnette [1970b], [1970e] Grünbaum [1970b], [1970c], [1970d]; compare below, §3.4).

Steinitz [1922] formulated his fundamental result not in terms of graphs, but in terms of 2-complexes. Since he was aware (see Steinitz [1922, p. 72]) of the fact that the graph of a 3-polytope determines the combinatorial type of the polytope, this is regrettable: the theorem is much easier to prove and to apply in the graph-theoretic formulation. Though Steinitz [1922] gives outlines of three different proofs, and though all three proofs are worked out in painstaking detail in Steinitz-Rademacher [1934], the theorem was not widely known until very recently.

One of Steinitz's proofs is reproduced in Lyusternik [1956]; unfortunately, Lyusternik's rendition (in the original, as well as in the two translations) is fallacious at a crucial stage of the proof, failing to present an adequate justification for one of the main steps (Theorem 2, p. 95, of the Russian original).

A detailed exposition of one of Steinitz's proofs of Theorem 1.8 (in terms of graphs) may be found in [CP, \$13.1]. Two additional and even simpler proofs are given in Barnette-Grünbaum [1969]. One of these proofs is analogous to another of Steinitz's methods, while the other is based on an idea of Kirkman [1857] which was 
independently rediscovered and greatly generalized by Tutte [1961]. In the special case of simple 3-polytopes (or dually, in the case of simplicial 3-polytopes) the proof of Steinitz's theorem may be greatly simplified; it was essentially known to Kirkman [1857] and Eberhard [1891], and was rediscovered by T. A. Brown [1960].

A variant, applicable to simple 3-polytopes having cyclically 4connected graphs, of one of the approaches to Steinitz's theorem for simple 3-polytopes was found by Kotzig [1970]. (A graph is said to be cyclically $n$-connected if the deletion of less than $n$ edges does not yield at least two components, each of which contains a circuit.) It is of special interest in connection with the search for simple 3-polytopes the graphs of which admit no Hamiltonian circuits (see below).

As another consequence of one of the methods of proof of Steinitz's theorem it is easy to show [CP, §13.2]:

THEOREM 1.9. Each 3-polytope may be approximated arbitrarily closely by 3-polytopes which have all their vertices at points having only rational coordinates in any given Cartesian system of coordinates for $E^{3}$.

(Compare below, $\$ 3.4$, for the contrasting situation in higher dimensions.)

Steinitz's theorem clearly implies that each 3-connected planar graph may be imbedded in the plane in such a fashion that all the bounded countries, as well as the complement of the (single) unbounded country, be convex polygons. Strengthenings of this result were independently obtained by Stein [1951] and Tutte [1960a], [1963]. (The author regrets the erroneous statement in Grünbaum [1967a, p. 291] that the proof of Stein [1951] is invalid.)

Another consequence of Steinitz's theorem (or of the results of Stein or Tutte) is the following theorem (Wagner [1936a], Fáry [1948], Stojaković [1959]; see also Woo [1969]):4

THEOREM 1.10. Every planar graph is geometrically imbeddable in the plane.

While the analogue of Theorem 1.10 for higher-dimensional complexes is false (see above, p. 18), the following two conjectures are open (compare Grünbaum [1967a, p. 253], [1969b], [1969d], Duke [1970]; see also Altschuler [1969]).

Conjecture 1.1. The topological imbeddability of a simplicial $k$ complex in $E^{2 k}$ implies its geometric imbeddability in $E^{2 k}$.

Conjecture 1.2. The topological imbeddability of a simplicial

${ }^{4}$ See Note 2 on page 1183. 
manifold in some Euclidean space implies its geometric imbeddability in the same space.

Using variants of the methods of proof devised by Steinitz rather than the final theorem, the graphs of 3-polytopes having a center (or a line, or a plane) of symmetry have been characterized ([CP, §13.2], Barnette [1970c]), ${ }^{5}$ and the following results established:

TheOREM 1.11. (Barnette-Griinbaum [1970].) The shape of any one 2-face of any 3-polytope may be arbitrarily prescribed.

Theorem 1.12. (Barnette [1970d].) If $P$ is a 3-polytope and if $D$ is a topological 2-disc which is the union of 2-faces of $P$, there exists a polytope $P^{\prime}$ and a point $x$ such that the part of $P^{\prime}$ visible from $x$ is precisely $D^{\prime}$, the union of the 2-faces of $P^{\prime}$ which correspond to the 2-faces of $P$ contained in D.

As a common generalization of the last two results we venture (compare Grünbaum [1970a]):

Conjecture 1.3. Let $P$ be a 3-polytope, let $C$ be a simple circuit in the graph of $P$ containing $n$ vertices, and let $F$ be a convex $n$-gon in a plane $L$. Then there exists a 3 -polytope $P^{\prime}$ such that

(i) $P^{\prime}$ is isomorphic to $P$;

(ii) The orthogonal projection $\pi$ of $E^{3}$ onto $L$ maps $P^{\prime}$ onto $F$;

(iii) The inverse image $\pi^{-1}$ (bd $F$ ) of the boundary of $F$ intersects $P^{\prime}$ precisely in the vertices and edges that correspond to $C$.

Analogues of Theorems 1.11 and 1.12 for higher-dimensional polytopes are not valid. For the former, an 8-dimensional counterexample is mentioned in Exercise 5.5.3 of [CP]; the validity of the theorem in dimensions 4 to 7 is still undecided. A 4-dimensional counterexample to the analogue of Theorem 1.12 may easily be derived from the complex TI of Grünbaum-Sreedharan [1967].

1.3. Eberhard's theorem. Among the easy consequences of Euler's theorem for 3-polytopes is the following fact concerning simple 3-polytopes:

Let $P$ be a simple 3-polytope and let $p_{k}(P)=p_{k}$ be the number of $k$-gonal faces of $P, k=3,4, \cdots$. Then

$$
\sum_{k \geq 3}(6-k) p_{k}=12 .
$$

This equation leads naturally to the question:

Given non-negative integers $p_{3}, \cdots, p_{n}$ satisfying $\sum_{k \geq 3}(6-k) p_{k}$ $=12$, does there exist a simple 3 -polytope $P$ such that $p_{k}(P)=p_{k}$ for $k=3, \cdots, n$ ?

\footnotetext{
S See Note 3 on page 1183 .
} 
It is easy to find examples (such as $p_{3}=4, p_{6}=1, p_{k}=0$ for $k \neq 3,6$ ) which show that the answer to the above question is negative. However, one may note that $p_{6}$ does not appear effectively in $(*)$, an observation which is put to good use in the following theorem:

THEOREM 1.13. (Eberhard [1891].) Given non-negative integers $p_{3}$, $p_{4}, p_{5}, p_{7}, \cdots, p_{n}$ satisfying (*), there exists a simple 3-polytope $P$ such that $p_{k}(P)=p_{k}$ for all $k \neq 6$.

Eberhard's [1891] proof of Theorem 1.13 is very complicated; a simpler proof may be found in [CP, §13.3], where Steinitz's Theorem 1.8 is used to reduce the proof of Eberhard's theorem to the construction of 3-valent, 3-connected planar graphs having the desired number of $k$-gonal faces for all $k \neq 6$.

Recently there has been considerable interest in ramifications, extensions, and analogues of Eberhard's theorem. We turn to an account of those results.

First, 4-valent 3-polytopes satisfy an equation analogous to (*), namely

$$
\sum_{k \geq 8}(4-k) p_{k}=8 .
$$

This suggests the following result similar to Eberhard's theorem, the proof of which is actually much simpler than that of Theorem 1.13 (see [CP, p. 254]):

THEOREM 1.14. Given non-negative integers $p_{3}, p_{5}, p_{8}, \cdots, p_{n}$ satisfying (**), there exists a 4-valent 3-polytope $P$ such that $p_{k}(P)=p_{k}$ for all $k \neq 4$.

A similar result holds for 2-arrangements, that is (see below, §3.1) for partitions of the projective plane by straight lines (Eberhard [1891], [CP, §18.2]).

It may be conjectured that analogues of Theorems 1.13 and 1.14 hold for 3-valent or 4-valent cell decompositions of any 2-manifold. However, even the case of the torus is only partially solved. Jucovič [1970c] and Zaks [1970a] have recently shown that the analogue of Theorem 1.14 holds for the torus (with the right-hand side of $(* *)$ equal to 0 ) for all sequences except $p_{3}=p_{5}=1, p_{k}=0$ for $k \geqq 6$.

Both proofs of Theorem 1.13 yield polytopes with large numbers of hexagons; moreover, although arbitrarily large numbers of hexagons may be incorporated in the construction, there is no control of the precise number. The situation changes if triangles and quadrangles are absent. Indeed, we have (Grünbaum [1968a]):

Theorem 1.15. If $p_{5}, p_{6}, p_{7}, \cdots, p_{n}$ are non-negative integers 
satisfying $\sum_{k \geq 5}(6-k) p_{k}=12$, and $p_{0} \geqq 8$, there exists a simple 3polytope $P$ such that $p_{k}(P)=p_{k}$ for all $k \geqq 5$.

It may be conjectured that 8 is the best possible bound in Theorem 1.15 .

The impossibility of dropping the assumption $p_{3}=p_{4}=0$, is demonstrated by the following results.

Theorem 1.16. There exist sequences of non-negative integers $p_{3}, p_{4}$, $p_{5}, p_{7}, \cdots, p_{n}$ satisfying $(*)$ such that the values of $p_{6}(P)$ possible in simple 3-polytopes $P$ with $p_{k}(P)=p_{k}$ for $k \neq 6$ omit infinitely many integers.

For example, if $p_{3}=4, p_{k}=0$ for $k \neq 3,6$, then $p_{0}$ must be even (Grünbaum-Motzkin [1963b]), while if $p_{3}=3, p_{4}=1, p_{5}=1, p_{k}=0$ for $k \geqq 7$, then $p_{6}$ must be odd and $\geqq 3$ [CP, p. 271]. (For additional results on related questions see $\$ 13.4$ of $[\mathrm{CP}]$ and the references given there, as well as Malkevitch [1969], [1970a], [1970b], Jucovič [1969], Grünbaum [1968a].)

TheOREM 1.17. (Barnette [1969a].) If $P$ is a 3-polytope (simple or $n o t)$ and if $\sum_{k \geq 7} p_{k}(P) \geqq 3$, then

$$
p_{6}(P) \geqq 2+\frac{p_{3}(P)}{2}-\frac{p_{5}(P)}{2}-\sum_{j \geq 7} p_{j}(P) .
$$

For another lower bound on $p_{6}$ see Jucovič [1970a].

From Theorem 1.17 follows a refutation of a conjecture in [CP, p. 268]. However, the following related conjecture (compare p. 267 and p. 269 of $[\mathrm{CP}]$ ) is still undecided:

For every sequence $p_{3}, p_{4}, p_{5}, p_{7}, p_{8}, \cdots, p_{n}$ satisfying $(*)$ there exists a simple 3-polytope $P$ such that $p_{k}(P)=p_{k}$ for $k \neq 6$, and

$$
p_{6}(K) \leqq 3 \sum_{k \geqq 3 ; k \neq 0} p_{k} .
$$

Let $v_{k}(P)$ denote the number of $k$-valent vertices of a 3-polytope $P$. Easy manipulations of Euler's formula yield, among others, the following analogues of $(*)$ and $(* *)$ :

$(* * * *)$

$$
\begin{gathered}
\sum_{i \geq 3}(6-i) p_{i}+2 \sum_{j \geq 3}(3-j) v_{j}=12, \\
\sum_{i \geq 3}(4-i)\left(p_{i}+v_{i}\right)=8 .
\end{gathered}
$$

A generalization of Theorem 1.14 established recently (Grünbaum [1969a]) is: 
TheOREM 1.18. Let $p_{3}, p_{5}, p_{6}, \cdots, p_{n}, v_{3}, v_{5}, v_{6}, \cdots, v_{m}$ be nonnegative integers satisfying (****) and such that $\sum_{i \geq 3} i_{i}$ is even. Then there exists a 3-polytope $P$ such that $p_{k}(P)=p_{k}$ and $v_{k}(P)=v_{k}$ for all $k \neq 4$.

It may be conjectured that a similar generalization of Theorem 1.13 holds for non-negative integers $p_{3}, p_{4}, p_{5}, p_{7}, \cdots, p_{n}, v_{4}, v_{5}, v_{6}, \cdots$, $v_{m}$ which satisfy $(* * *)$.

For a somewhat related result see Jucovič [1970b]. ${ }^{6}$

Generalizing the ideas involved in Eberhard's theorem in a different direction, the following analogue of Theorem 1.13 was recently established by Rowland [1968] (along with the corresponding generalizing of Theorem 1.14).

THEOREM 1.19. If $p_{1}, \cdots, p_{5}, p_{7}, \cdots, p_{n}$ are non-negative integers satisfying

$$
\sum_{k \geq 1}(6-k) p_{k}=12,
$$

there exists a 3-valent connected "graph" (possibly with loops and 2circuits) in the plane such that the number of $k$-gonal countries it determines equals $p_{k}$ for all $k \neq 6$.

Similar generalizations probably hold for all 2-manifolds. ${ }^{7}$

Reformulating Eberhard's theorem or the other results mentioned above for the dual graphs or polytopes, one obtains statements concerning the valences of vertices of graphs which triangulate the 2sphere. Then it is rather immediate to ask about the valences of the vertices in graphs which are not assumed to triangulate the plane, or to be planar, or which are restricted by some other requirement. As a matter of fact, such topics were the object of a considerable number of papers; see Grünbaum [1970f] for a detailed survey and for references. Here we shall mention only two aspects:

First, the open problem (Hakimi [1962], Böttger-Harders [1964l. Anonymous [1964], Chvátal [1969]) of characterizing the sequences $\left(a_{1}, \cdots, a_{n}\right)$ which may occur as the valences of vertices of planar graphs.

Second, a theorem which is typical of the more general results of Gallai [1970] (which extends earlier work of Motzkin [1964], [1967a], [1967b], Kotzig [1965], [CP, \$13.4], Heawood [1936], Malkevitch [1969], [1970a], Dalkey [1967]). In the theorem $s$ and $t$

"See Note 4 on page 1183.

${ }^{7}$ See Note 5 on page 1183. 
are integers such that $s, t \geqq 3$ and $(s-2)(t-2)<4$; we denote by $\pi(s, t)$ the number of faces ( $s$-gons) of the regular (=Platonic) solid $\{s, t\}$, and we designate as an $s$-angulation of the sphere every decomposition of the 2-sphere into $s$-gons.

THEOREM 1.20. If the valence of each vertex of an s-angulation of the sphere is a multiple of $t$, then the number of $s$-gons is a multiple of $\pi(s, t)$.

For some related questions see Wagner [1936b], Grötzsch [1956], [1962], Hauschild [1967], [1968], Hawkins-Hill-Reeve-Tyrrell [1966], Meek [1968], Chvátal [1969], Crowe-Molnár [1969], and Crowe [1969].

1.4. Paths and trees on 3-polytopes. The general question whether each member of a certain class of graphs (for example, $d$-polytopal graphs) contains a subgraph of a specified type (for example, a Hamiltonian circuit, or a subdivision of a complete graph, etc.) arises in contexts as various as coding theory, linear programming, the four-color problem, or the nomenclature of organic compounds. The results obtained and methods used vary as greatly as the motivating problems. We already mentioned in $\$ 1.1$ some results of this kind; a discussion of some related areas (partly overlapping with the following exposition) may be found in Chapters 16 and 17 (written by Victor Klee) of Grünbaum [1967a]. Here we shall restrict our attention mainly to 3-polytopes, and only to a few of the relevant problems.

We first consider Hamiltonian circuits [and paths] on graphs of simple 3-polytopes (that is, simple circuits [or paths] containing all the vertices). Tait [1880] conjectured in 1880 that the graph of each simple 3-polytope admits a Hamiltonian circuit; it is easy to verify that if such a graph possesses a Hamiltonian circuit then the countries of the corresponding map (that is, the 2-faces of the 3-polytope) are colorable with 4 colors. (For a result concerning the coloring of 2-faces of higher-dimensional polytopes see Grünbaum [1970g].) Claims of proof of Tait's conjecture have been made by Schoblik [1930] and Chuard [1932] (in 1930 and 1932); however, in 1946 Tutte [1946] found a counterexample (with 46 vertices) to Tait's conjecture. Motivated by the 4-color problem, there was interest in deciding Tait's conjecture for graphs of simple 3-polytopes which are, moreover, cyclically 4- or 5-connected. The first example of a cyclically 4-connected 3-valent 3-connected planar graph without a Hamiltonian circuit was given by Tutte [1960b]; a smaller such graph (58 vertices) was found by Hunter [1962]. An example of a cyclically 5-connected graph with these properties was first found by Walther 
[1965]; Walther's graph has 162 vertices. (See also Walther [1966], [1968], [1969a].) The smallest known examples of graphs of simple 3 -polytopes without Hamiltonian circuits are shown in Figures 1, 2 and 3; they are cyclically 3-, 4-, and 5-connected, and have 38, 42, and 44 vertices, respectively. The first of them was independently found by Lederberg [1966], Bosák [1967], and D. Barnette (private communication). The second is easy to find using the following Theorem 1.21 due to Grinberg [1968] and Sachs [1968]; the graph of Figure 3 is due to W. T. Tutte (private communication) and has two vertices less than a similar graph given by Sachs [1968].

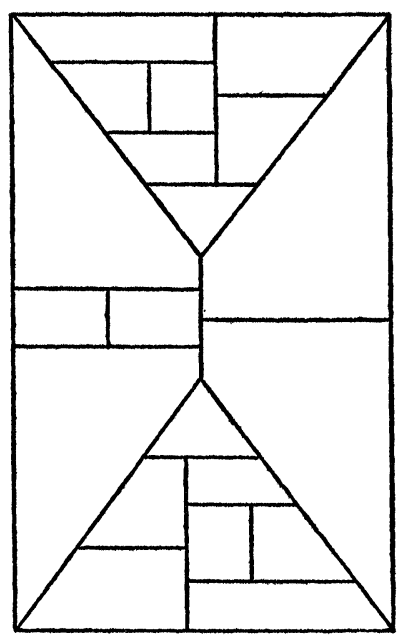

Figure 1

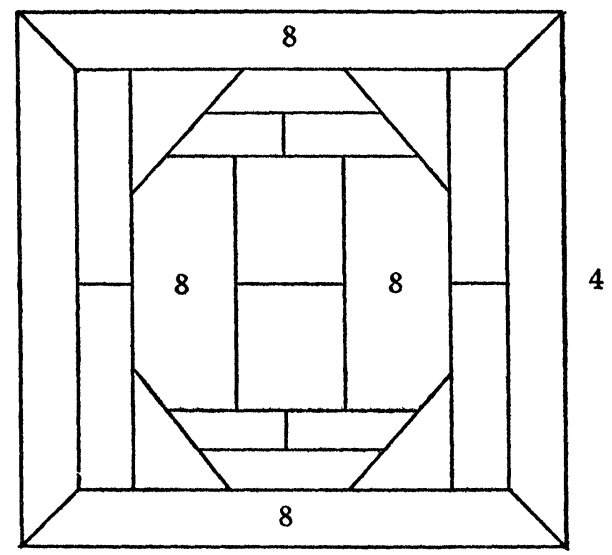

FigURE 2 


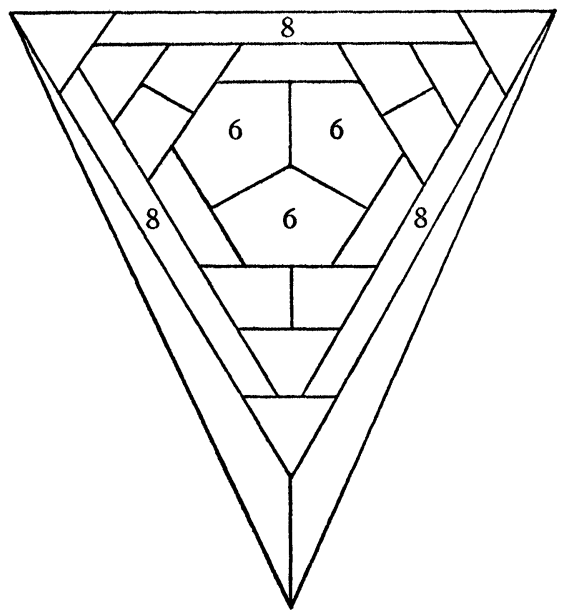

Figure 3

(Due to W. T. Tutte)

Theorem 1.21. If a simple 3-polytope $P$ admits a Hamiltonian circuit, then there exists a decomposition $p_{k}(P)=p_{k}^{\prime}+p_{k}^{\prime \prime}$ (with nonnegative integers $\left.p_{k}^{\prime}, p_{k}^{\prime \prime}\right)$ such that

$$
\sum_{k \geq 3}(k-2) p_{k}^{\prime}=\sum_{k \geq 3}(k-2) p_{k}^{\prime \prime} .
$$

Taking this equation modulo 3 , it easily follows that the graphs of Figures 2 (in which all 2-faces but one are either pentagons or octagons) and 3 admit no Hamiltonian circuit.

The graphs of Figures 1, 2, and 3 are probably the smallest examples of the respective types. This, however, is far from established; indeed, it is only known (Lederberg [1967]) that the graph of each simple 3-polytope with at most 18 vertices has a Hamiltonian circuit. A number of related problems was solved by Barnette-Jucovič [1970].

Two conjectures due to David Barnette should be recorded in this context.

Conjecture 1.4. If all 2-faces of a simple 3-polytope $P$ are polygons with an even number of edges, then the graph of $P$ has a Hamiltonian circuit.

Conjecture 1.5. The graph of each simple 4-polytope has a Hamiltonian circuit.

Conjecture 1.5 has not been settled even for 4-dimensional prisms having simple 3-polytopes as bases.

It is worth mentioning that the analogue of Conjecture 1.4 in which all of the polygons of $P$ are assumed to have a number of edges 
divisible by 3 has a negative solution. Indeed, by "cutting off" suitable sets of vertices in each of the non-Hamiltonian graphs mentioned above (that is, replacing each chosen vertex by a "small" triangle) it is easy to construct non-Hamiltonian graphs such that the number of edges of each country is a multiple of 3 .

Conjecture 1.5 is precariously balanced between two known facts:

Theorem 1.22. (Grünbaum-Motzkin [1962], Moon-Moser [1963].) There exist, for every $d \geqq 3$, d-polytopal graphs which have no Hamiltonian circuit.

Theorem 1.23. (Tutte [1956]; see also Ore [1967, Chapter 5.2].) Each 4-connected planar graph admits a Hamiltonian circuit.

In this context we have

Conjecture 1.6. Each 4-connected graph embeddable in the torus, or in the projective plane, admits a Hamiltonian circuit.

Probably there exists a function $c(n)$ such that every $c(n)$-connected graph embeddable in a surface of genus $\leqq n$ admits a Hamiltonian circuit. $^{8}$

Very little is known concerning higher-dimensional analogues of Hamiltonian circuits. The possibility of fruitfully exploring and exploiting this area has been recently demonstrated by Barnette [1969b].

We turn now to the topic of Hamiltonian paths and longest simple paths in polytopal graphs; it is of interest in connection with existence problems of certain types of higher-dimension polytopes (see below, §2.2). We mention the following sample results.

THEOREM 1.24. There exists a simple 3-polytope with 88 verlices which allows no Hamiltonian path.

Figure 4 shows a graph of this type, kindly communicated to the author by Tudor Zamfirescu.

THEOREM 1.25. There exist constants $\alpha<1$ and $c$, and simple 3polytopes $P_{n}$ with $n$ vertices such that the longest simple path in $P_{n}$ contains less than $\mathrm{cn}^{\alpha}$ vertices of $P_{n}$.

Theorem 1.25 was established in Grünbaum-Motzkin [1962], with $c=2$ and $\alpha=1-2^{-19}$; recently $\mathrm{R}$. Forcade (private communication) using modified arguments improved those bounds to $c=3 / 2, \alpha=1$ $-2^{-11}$. An independent proof of Theorem 1.25 for circuits was given by Walther [1967]. The best result in this direction is due to $H$. Walther (private communication). He established Theorem 1.25 with

${ }^{8}$ See Note 6 on page 1183. 


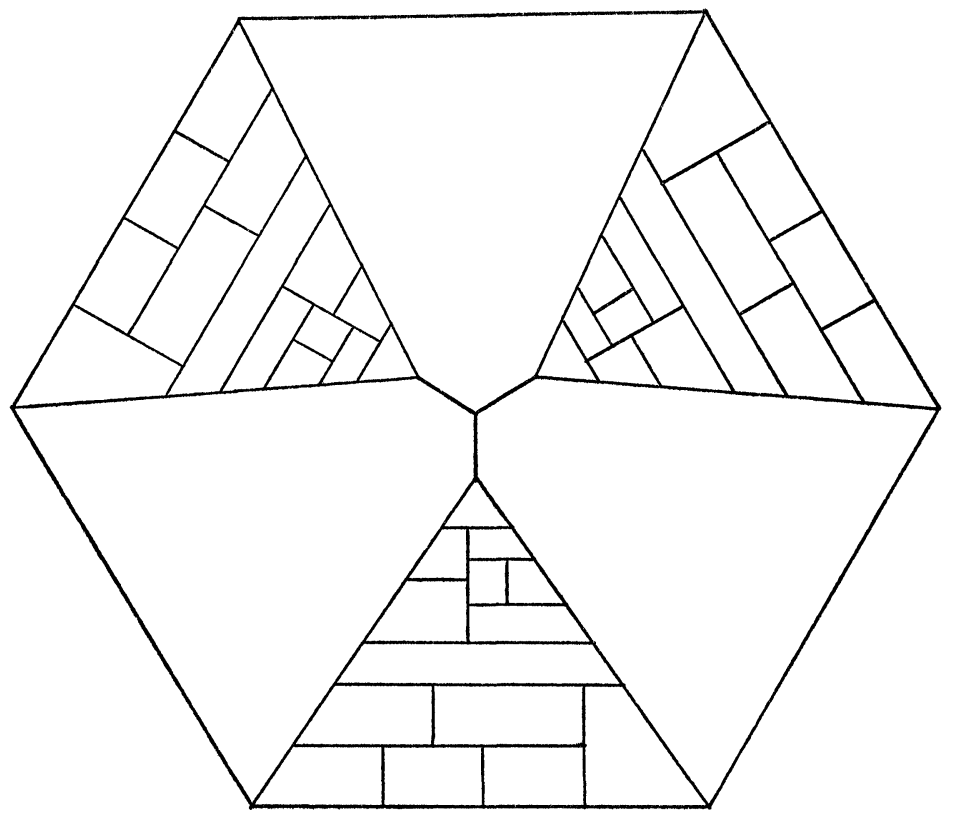

Figure 4

(Due to T. Zamfirescu)

$c=2$ and $\alpha<1-2^{-7}$, even under the additional condition that all the faces of $P_{n}$ be polygons with at most 21 sides.

For an inconclusive discussion of Hamilton circuits, and 2-factors, in 3-valent planar graphs see Chuard [1966].

Simple paths on 3-polytopal graphs (corresponding to not necessarily simple polytopes) have been investigated by $\mathrm{T}$. A. Brown [1961], Moon-Moser [1963] and others (see $\$ 17.2$ of [CP]); see also Walther [1969b], Klee-Walkup [1967], Jucovič [1968], and Larman [1970].

Another interesting result concerning graphs of 3-polytopes is due to Barnette [1966]; we recall that a graph $T$ is called a spanning tree of a graph $G$ provided:

(i) $T$ is a tree;

(ii) $T$ is a subgraph of $G$; and

(iii) each vertex of $G$ belongs to $T$.

Barnette's result is

THEOREM 1.26. Each 3-polytopal graph admits a spanning tree of maximal valence 3.

It may be conjectured that each 3-polytopal graph $G$ admits a 
spanning $T$ of maximal valence 3 , with the property that the edges of the dual graph $G^{*}$ which correspond to the edges of $G$ not in $T$, form a spanning tree of $G^{*}$ of maximal valence 3 .

2. Euler's relation; combinatorial and other invariants. In the present section we shall survey the relations known to exist between the numbers of faces of various dimensions of a $d$-polytope, as well as similar relations concerning other entities associated with polytopes.

2.1. f-vectors. For a $d$-polytope $P$ [or for a $(d-1)$-complex $\mathfrak{C}]$ we shall call $f$-vector of $P$ [or $\mathfrak{e}$ ] the $d$-dimensional vector $f(P)$ $=\left(f_{0}(P), \cdots, f_{d-1}(P)\right)\left[\right.$ or $\left.f(\mathbb{C})=\left(f_{0}(\mathfrak{C}), \cdots, f_{d-1}(\mathbb{C})\right)\right]$, where $f_{k}(P)$ is the number of $k$-faces of $P$ for $-1 \leqq k \leqq d-1$. We also find it convenient to adopt the convention $f_{d}(P)=1$ and $f_{i}(P)=0$ for $i<-1$ or $i>d$. For a family $\rho$ of $d$-polytopes we put $f(\rho)=\{f(P) \mid P \in \odot\}$ and $A(P)=\operatorname{aff}(f(P))$, where aff $K$ denotes the affine hull of the set $K$. We shall use a similar notation for families of complexes.

Clearly, the characterization of $f(P)$ for various families $\beta$ of polytopes is an important step in the investigation of the structure of members of $P$. We shall review in this subsection mainly the results known in case $\odot$ is $\odot^{d}$, the family of all $d$-polytopes, or $\mathcal{P}_{s}^{d}$, the family of all simplicial $d$-polytopes.

The only non-trivial case in which $f\left(\odot^{d}\right)$ has been completely characterized concerns $d=3$. We have

Theorem 2.1. (Steinitz [1906].) $\left(f_{0}, f_{1}, f_{2}\right) \in f\left(\odot^{3}\right)$ if and only if the integers $f_{0}, f_{1}, f_{2}$ satisfy

$$
f_{1}=f_{0}+f_{2}-2, \quad 4 \leqq f_{0} \leqq 2 f_{2}-4, \quad 4 \leqq f_{2} \leqq 2 f_{0}-4 .
$$

The similar characterization of $f\left(\odot_{s}^{3}\right)$ is quite trivial: $\left(f_{0}, f_{1}, f_{2}\right)$ $\in f\left(\odot_{s}^{3}\right)$ if and only if $f_{0} \geqq 4, f_{1}=3 f_{0}-6, f_{2}=2 f_{0}-4$.

In higher dimensions only the flats $A\left({\odot^{d}}^{d}\right)$ and $A\left({\odot_{s}^{d}}^{d}\right)$ have been characterized.

THEOREM 2.2. (Euler.) The dimension of $A\left(\rho^{d}\right)$ is $d-1$; more precisely,

$$
A\left(\odot^{d}\right)=\left\{\left(f_{0}, \cdots, f_{d-1}\right) \mid \sum_{i=0}^{d-1}(-1)^{i} f_{i}=1-(-1)^{d}\right\} .
$$

THEOREM 2.3. (Dehn-Sommerville.) The dimension of $A\left(\odot_{s}^{d}\right)$ is $[d / 2]$; more precisely, $A\left(\odot_{s}^{d}\right)$ may be represented as the intersection of the hyperplanes determined by ihe Dehn-Sommerville equations

$$
\sum_{j=k}^{d-1}(-1)^{j}\left(\begin{array}{l}
j+1 \\
k+1
\end{array}\right) f_{j}=(-1)^{d-1} f_{k} \text { for }=-1,0,1, \cdots, d-1 .
$$


It is easily checked that the equations corresponding to $k=d-2$, $d-4, d-6, \cdots$ form an independent system of $\left[\frac{1}{2}(d+1)\right]=d$ $-[d / 2]$ equations. With minor modifications, the equations are valid for all simplicial manifolds, and even for more general types of simplicial complexes.

References to the proofs and to the long and involved history of those results and of related questions concerning various families of polytopes and complexes may be found in Grünbaum [1967a], [1967b], Klee [1966], and Grünbaum-Shephard [1969]. To those references one should add the early and remarkable - though practically forgotten-paper of Vaccaro [1956] (see also Vaccaro [1954]). Vaccaro not only established Theorem 2.3 for simplicial manifolds, but was also the first one to use explicitly the notion of $f$-vectors. Another independent approach is that of Hadwiger [1968]. ${ }^{9}$

For a generating-functions approach which works equally well for the Dehn-Sommerville equations for simplicial polytopes (and their analogs for other families) and for the corresponding angle-sums relations (see §2.2) see Macdonald [1970], McMullen-Shephard [1968b].

It is rather easily seen that, if $n=\left[\frac{1}{2} d\right]$, then $f_{0}, \cdots, f_{n-1}$ may be chosen as independent parameters for the solutions of the DehnSommerville equations. In other words, we may write

$$
f_{n+k}=\sum_{i=0}^{n-1} \alpha_{i}(d, k) f_{i} \text { for } d=2 n \text { and } k=0, \cdots, n-1,
$$

and

(**) $f_{n+k}=\beta_{-1}(d, k)+\sum_{i=0}^{n-1} \beta_{i}(d, k) f_{i}$ for $d=2 n+1$ and $k=0, \cdots, n$.

For example,

$$
\begin{aligned}
& \alpha_{i}(2 n, n-1)=(-1)^{n+i+1} \frac{i+1}{n}\left(\begin{array}{c}
2 n-2-i \\
n-1
\end{array}\right), \\
& \beta_{i}(2 n+1, n)=(-1)^{n+i+1} 2\left(\begin{array}{c}
2 n-1-i \\
n
\end{array}\right), \text { etc. }
\end{aligned}
$$

The expression for $\alpha_{i}(d, k)$ and $\beta_{i}(d, k)$ in the general case do not seem to be representable in closed form with the help of the usual functions of combinatorial analysis (see Grünbaum [1967a, p. 160], Riordan [1966]). However, starting from expressions obtained by Macdonald [1970], McMullen [1970a] has recently established:

- See Note 7 on page 1183. 
TheOREM 2.4. For $0 \leqq k<n$ and $0 \leqq i \leqq n-1$,

$$
\alpha_{i}(2 n, k)=(-1)^{n+i+1} \frac{i+1}{n+k-i} \sum_{j=0}^{n-1}\left(\begin{array}{c}
n-1-j \\
k
\end{array}\right)\left(\begin{array}{c}
2 n-1-i-j \\
n
\end{array}\right) \text {, }
$$

while, for $0 \leqq k \leqq n$ and $-1 \leqq i \leqq n-1$,

$$
\beta_{i}(2 n+1, k)=(-1)^{n+i+1} \frac{n+k+2}{n+k-i} \sum_{j=0}^{n}\left(\begin{array}{c}
n-j \\
k
\end{array}\right)\left(\begin{array}{c}
2 n-i-j \\
n+1
\end{array}\right) .
$$

The formulae of McMullen have one advantage over the previously known expressions for the $\alpha_{i}$ and $\beta_{i}$; they show that the coefficients in $\left(^{*}\right)$ and $\left({ }^{*}\right)$ have alternating signs. This fact has been used by McMullen [1970a] to settle certain cases of the so-called "upperbound conjecture," which is the next topic of our discussion. ${ }^{10}$

For integers $d, k, v$ satisfying $1 \leqq k \leqq d-1, v \geqq d+1$, the "upperbound conjecture" $\mathrm{UBC}(d, k ; v)$ may be formulated as follows:

$\operatorname{UBC}(d, k ; v)$. The cyclic $d$-polytopes with $v$ vertices have the maximal number of $k$-faces among all $d$-polytopes with $v$ vertices.

We recall that a cyclic $d$-polytope $C(v, d)$ with $v$ vertices is defined as the convex hull of any $v$ different points of the moment curve $\left\{\left(t, t^{2}, \cdots, t^{d}\right) \mid-\infty<t<\infty\right\}$ in the $d$-space. Cyclic polytopes were first considered by Carathéodory [1907] in 1907; they were rediscovered about half a century later by D. Gale and T. S. Motzkin (see Grünbaum [1967a, §4.7] for details and references). ${ }^{11}$ The numbers $f_{k}(C(v, d)$ ) are known (Grünbaum [1967a, §9.6], McMullenShephard [1968b]); extending an elegant result of Gale [1963], Shephard [1968d] has recently characterized sets of vertices of $C(v, d)$ which determine $k$-faces of $C(v, d)$, and provided simple arguments for the determination of the numbers $f_{k}(C(v, d))$. We mention explicitly

$$
\begin{gathered}
f_{k}(C(v, d))=\left(\begin{array}{c}
v \\
k+1
\end{array}\right) \text { for } 0 \leqq k<[d / 2] . \\
f_{n}(C(v, d))=\left(\begin{array}{c}
v \\
n+1
\end{array}\right)-\left(\begin{array}{c}
v-n-2 \\
n
\end{array}\right) \frac{v}{n+1} \quad \text { for } d=2 n \\
=\left(\begin{array}{c}
v \\
n+1
\end{array}\right)-\left(\begin{array}{c}
v-n-2 \\
n+1
\end{array}\right) \quad \text { for } d=2 n+1,
\end{gathered}
$$

and

10 See Note 8 on page 1183.

11 See Note 9 on page 1184. 


$$
\begin{aligned}
f_{d-1}(C(v, d)) & =\left(\begin{array}{c}
v-n \\
n
\end{array}\right) \frac{v}{v-n} \text { for } d=2 n, \\
& =2\left(\begin{array}{c}
v-n-1 \\
n
\end{array}\right) \text { for } d=2 n+1 ;
\end{aligned}
$$

the expressions are more involved for other values of $k$.

For various reasons, the most interesting cases of the upper-bound conjecture are those in which $k=d-1$. Though $\mathrm{UBC}(d, d-1 ; v)$ has not been completely established, it has been proved in "almost all" cases, due mainly to the pioneering work of Klee [1964b], [1964c]. We have

THEOREM 2.5. In each of the following cases the maximal number $\mu(v, d)$ of $(d-1)$-faces for $d$-polytopes with $v$ vertices is attained by the cyclic polytopes $C(v, d)$ :

(i) $d \leqq 8$, v arbitrary;

(ii) $d$ arbitrary, $v \leqq d+3$;

(iii) $d$ arbitrary, $v \geqq n^{2}-2$ for even $d=2 n$, or $v \geqq n^{2}+2 n-2$ for odd $d=2 n+1$.

Hence the $\operatorname{UBC}(d, d-1, v)$ is still in doubt only for finitely many values of $v$ in each dimension $d \geqq 9$. For $d=9$ the undecided cases are $13 \leqq v \leqq 21$.

Proofs of the various parts of Theorem 2.5 and of the analogous results on the upper-bound conjecture for $k<d-1$, with references to the rather voluminous literature, may be found in Grünbaum [1967a, \$10.1] and in McMullen-Shephard [1968b]. Only the subcase of (iii) in which $v$ has the least allowed value is more recent and not mentioned in [CP]; its proof is given in Grünbaum [1969c].

For proofs of the upper-bound conjecture for $k<d-1$ in some cases not covered in [CP] see Grünbaum [1969c] and McMullen [1970a], [1970b].12

All the proofs of special cases of the upper-bound conjecture rely on certain non-linear relations between the $f_{i}$ 's. Such relations are also of independent interest, as they provide additional information on the sets $f\left(\rho_{s}^{d}\right)$, and lead to many open problems. Some relations of this type are given in the theorems that follow.

Theorem 2.6. (i) (Klee [1964c], Grïnbaum [1967a].) For $0 \leqq r \leqq k$,

$$
\left(\begin{array}{c}
k+1 \\
r
\end{array}\right) f_{k} \leqq\left(\begin{array}{c}
f_{0}+r-1-k \\
r
\end{array}\right) f_{k-r} .
$$

12 See Note 8 on page 1183. 
(ii) (Grünbaum [1970c].) For $0 \leqq r \leqq k$,

$$
(k+1)\left(\begin{array}{l}
k \\
r
\end{array}\right) f_{k} \leqq\left(\begin{array}{c}
f_{0}+r-k \\
r
\end{array}\right)\left((k+1-r) f_{k-r}-r f_{k-r-1}\right) .
$$

Both parts of Theorem 2.6 are valid for complexes. However, while (i) is known to hold for the $f$-vectors of any topological cell complex, the validity of (ii) has been established only for simplicial complexes (and for cell complexes if $k=2$ ). This does not affect the usefulness of (ii) in connection with the upper-bound conjecture, because of the lower semicontinuity of $f_{k}(P)$ as a function of $P$ (see Eggleston-Grünbaum-Klee [1964], or [CP]). Even the validity of (ii) for non-simplicial polytopes is still undecided if $k \geqq 3$.

A very elegant result is due to Kruskal [1963] and Katona [1968]; it may be formulated as follows:

Theorem 2.7. Let $\mathfrak{e}$ be a simplicial complex and let

$$
f_{k}(\mathcal{C})=\left(\begin{array}{c}
a_{k+1} \\
k+1
\end{array}\right)+\left(\begin{array}{c}
a_{k} \\
k
\end{array}\right)+\left(\begin{array}{c}
a_{k-1} \\
k-1
\end{array}\right)+\cdots+\left(\begin{array}{c}
a_{i} \\
i
\end{array}\right)
$$

where $i \geqq 1, a_{k+1}>a_{k}>\cdots>a_{i}$, and $a_{j} \geqq j$ for $j=i, \cdots, k+1$. (Such a representation always exists and is unique.) Then the number of $p$ faces of $\mathfrak{e}$, where $p<j[$ respectively $p>k]$, is at least [at most]

$$
\left(\begin{array}{c}
a_{k+1} \\
p+1
\end{array}\right)+\left(\begin{array}{c}
a_{k} \\
p
\end{array}\right)+\left(\begin{array}{c}
a_{k-1} \\
p-1
\end{array}\right)+\cdots \cdot
$$

For some related results see Hansel [1967].

The Kruskal-Katona Theorem 2.7 may be used to settle certain cases of the upper-bound conjecture which are not tractable by other methods presently available (see Grünbaum [1967a], [1969c], McMullen [1970b]).

Contrasting the upper-bound conjecture is a pair of problems which seem to be very difficult: The determination of the lower bounds $\phi_{k}(v, d)$ and $\phi_{k}^{*}(v, d)$ of $f_{k}(P)$ for polytopes $P$ such that $f_{0}(P)=v$ and $P \in \mathcal{P}^{d}$ or $P \in \mathcal{P}_{s}^{d}$. Concerning the first problem, for $d \geqq 4$ the values of $\phi_{k}(v, d)$ are known only if $v \leqq d+4$.

We have (Grünbaum [1967a]):

THEOREM 2.8 . For $1 \leqq k \leqq d-1$ and $5 \leqq d+1 \leqq v \leqq d+4$,

$$
\phi_{k}(v, d)=\left(\begin{array}{l}
d+1 \\
k+1
\end{array}\right)+\left(\begin{array}{c}
d \\
k+1
\end{array}\right)-\left(\begin{array}{c}
2 d+1-v \\
k+1
\end{array}\right) \text {. }
$$

It has been conjectured that Theorem 2.8 is valid for all $v \leqq 2 d$; for $v>2 d$ there seems to be neither a conjecture about the value of 
$\phi_{k}(v, d)$, nor any guess as to which $d$-polytopes $P$ satisfy $f_{0}(P)=v$, $f_{k}(P)=\phi_{k}(v, d)$.

In the special case $k=d-1$ remarkable additional results were obtained by McMullen [1970c]; they correct and extend Theorem 10.2.3 of Grünbaum [1967a]. For the formulation of McMullen's results we recall (see Theorem 2.5 ) that $\mu(v, d)$ denotes the maximal possible number of $(d-1)$-faces in a $d$-polytope with $v$ vertices, and we define a new function $\lambda(v, d)$ as follows:

If $d=2 n+1$ is odd, and if for some $w \geqq d+3$ and some $1 \leqq k \leqq[n / 2]$ we have $v=\mu(w, d)+1-2 k$, then $\lambda(v, d)=w+1$.

If $d+2 \leqq v \leqq\left[(d+2)^{2} / 4\right]$ and $v$ is not of the form $v=r+(s+1)(t+1)$ for some integers $r \geqq 0, s \geqq 1, t \geqq 1$ such that $r+s+t=d$, then $\lambda(v, d)$ $=2 n+4$, where $n=[d / 2]$.

If $v$ and $d$ are not of either of the above types, then

$$
\lambda(v, d)=\min \{w \mid \mu(w, d) \geqq v\} .
$$

McMullen [1970c] made the following "lower-bound conjecture": $\operatorname{LBC}(d ; v)$. For all $v>d \geqq 2, \phi_{d-1}(v, d)=\lambda(v, d)$.

The following two results were established by McMullen [1970c].

THEOREM 2.9. $\phi_{d-1}(v, d) \geqq \lambda(v, d)$ for all $v>d \geqq 2$.

TheOREM 2.10. The $\operatorname{LBC}(d ; v)$ holds if $d \leqq 4$, or if $v \leqq[d(d+8) / 4]$, or if $v=\mu(w, d)+t$ for some $w \geqq d+2$ and some $t$ such that $0 \leqq t \leqq d-1$ for even $d$, or $-1 \leqq i \leqq d-1$ for odd $d$.

For additional related results see McMullen [1970d].

Concerning $\phi_{k}^{*}(v, d)$ it has been frequently conjectured that

$$
\phi_{k}^{*}(v, d)=\left(\begin{array}{l}
d \\
k
\end{array}\right) v-\left(\begin{array}{l}
d+1 \\
k+1
\end{array}\right) k \text { for } 1 \leqq k \leqq d-2
$$

and

$$
\phi_{d-1}^{*}(v, d)=(d-1) v-(d+1)(d-2),
$$

and that the lower bound is attained for a well-determined family of simplicial $d$-polytopes. The problem is known as "simplicial lowerbound conjecture." While it is easy to prove the simplicial lowerbound conjecture if either $d \leqq 3$, or else $v \leqq d+3$ (see Grünbaum [1967a, \$10.2]), non-trivial results were obtained only very recently. Walkup [1970] proved, among related results on triangulations of manifolds,

THEOREM 2.11. The simplicial lower-bound conjecture is true if $d=4$ or $d=5$. 
M. A. Perles, in unpublished work, established

THEOREM 2.12. The simplicial lower-bound conjecture is true for $v \leqq d+10$.

Perles also established that the estimate $f_{k}(P) \geqq \phi_{k}^{*}(v, d)$ is valid if $v \leqq d+11$, but was unable to characterize the simplicial $d$-polytopes $P$ for which equality holds.

There is an additional recent development that deserves attention. ${ }^{13}$ Following McMullen-Walkup [1970] let

$$
g_{k}=\sum_{j=-1}^{k}(-1)^{k-j}\left(\begin{array}{l}
d-j \\
d-k
\end{array}\right) f_{j} .
$$

Then

$$
f_{j}=\sum_{k=-1}^{j}\left(\begin{array}{l}
d-k \\
d-j
\end{array}\right) g_{k},
$$

and the Dehn-Sommerville equations of Theorem 2.3 are easily seen to be equivalent to the system

$$
g_{k}=-g_{d-k-1} \text { for }-1 \leqq k \leqq[(d-1) / 2] .
$$

McMullen-Walkup [1970] made the following "generalized lowerbound conjecture for simplicial $d$-polytopes":

$$
g_{k} \geqq 0 \quad \text { for }-1 \leqq k<[d / 2] .
$$

It may be noted that the cases $k \leqq 0$ of the conjecture are trivial; the case $k=1$ reduces for $d \geqq 4$ to the conjecture

$$
\phi_{1}^{*}(v, d)=d v-\left(\begin{array}{c}
d+2 \\
2
\end{array}\right)
$$

mentioned above, and is by Theorem 2.11 valid for $d=4$ and $d=5 .{ }^{14}$

The above results may be interpreted as information on the shape of the projection of $f\left(\odot^{d}\right)$ or $f\left(\odot_{s}^{d}\right)$ onto certain coordinate subspaces. In a few special cases, more is known about those projections. For example, the sets $\left\{\left(f_{0}(P), f_{1}(P)\right) \mid P \in \mathcal{P}^{4}\right\}$ and $\left\{\left(f_{0}(P), f_{3}(P)\right) \mid P \in \mathcal{P}^{4}\right\}$ have been completely characterized (Grünbaum [1967a, \$10.4]), while the sets $\left\{\left(f_{0}(P), f_{2}(P)\right) \mid P \in \mathcal{P}^{4}\right\}$ and $\left\{\left(f_{1}(P), f_{2}(P)\right) \mid P \in \mathcal{P}^{4}\right\}$ were investigated by Reay [1968a] and Barnette-Reay (unpub- 
lished). In the first of the above cases the most interesting feature is the non-existence of 4-polytopes corresponding to two "exceptional" pairs $(8,17)$, and $(10,20)$, which are not excluded by any general condition known. (The pairs $(6,12)$ and $(7,14)$ are excluded by Theorem 2.8; they are analogous to the well-known non-existence of 3 -polytopes with precisely 7 edges.) Similar "exceptions" are probably to be expected in most projections of the sets $f\left(\odot^{d}\right)$.

Many additional problems concerning the $f$-vectors of polytopes or complexes of various types still remain to be solvcd, or even to be properly posed. One question of such nature concerns inequalities between the $f_{k}$ 's for complexes $\mathbb{e}$ embeddable in a space of given dimension. We mention only one result of this type, which is very easy to prove:

Theorem 2.13. If $\mathfrak{e}$ is a 2-dimensional topological cell complex in $E^{3}$ such that each vertex and each edge belongs to at least two 2-faces of $\mathfrak{C}$ then

$$
f_{2}(\mathbb{C}) \leqq 2 f_{1}(\mathbb{C})-2 f_{0}(\mathbb{C}) .
$$

For a proof of Theorem 2.13 and some generalizations, and for an application of such inequalities to coloring problems on polytopes (analogous to the four-color conjecture) see Grünbaum [1970g].

The remark that the inequality of Theorem 2.13 becomes an equation if $\mathfrak{C}$ is the 2 -skeleton of a simplicial 4-polytope motivates the following

CONJECTURe 2.1. For each $n$-complex $\mathfrak{e}$ such that each $i$-face, $0 \leqq i \leqq n-1$, of $\mathcal{C}$ belongs to at least $n n$-faces of $\mathfrak{C}$ we have

$$
f_{n}(\mathbb{C}) \leqq \sum_{i=0}^{n-1}(-1)^{n+i+1} \frac{i+1}{n+1}\left(\begin{array}{c}
2 n-i \\
n
\end{array}\right) f_{i}(\mathbb{C})
$$

if $\mathcal{C}$ is embeddable in $E^{2 n-1}$, and

$$
f_{n}(\mathfrak{e}) \leqq(-1)^{n} 2\left(\begin{array}{c}
2 n+1 \\
n
\end{array}\right)+\sum_{i=0}^{n-1}(-1)^{n+i+1}\left(\begin{array}{c}
2 n+1-i \\
n+1
\end{array}\right) f_{i}(\mathfrak{e})
$$

if $\mathfrak{C}$ is embeddable in $E^{2 n}$.

The first part of the conjecture is open for all $n \geqq 3$, the second for all $n \geqq 2$; it would be interesting to decide the conjecture even in the special case that $\mathfrak{C}$ is simplicial.

A number of additional classes of $d$-polytopes have been considered. We mention here only 
(i) cubical polytopes (and complexes), all proper faces of which are (combinatorial) cubes;

(ii) self-dual polytopes (i.e. $d$-polytopes combinatorically equivalent to their duals);

(iii) centrally symmetric polytopes.

For each of those three classes an analog of Theorem 2.3 is valid (see Grünbaum [1967a, Chapter 9]), and problems analogous to the upper- and lower-bound conjectures may be posed. Among the few known results concerning those questions we mention only that for centrally symmetric $d$-polytopes with $f_{0}$ vertices the upper bounds for $f_{k}$ are smaller than the value obtained from Theorem 2.5 and the requirement that all $f_{i}$ 's be even (Grünbaum [1967a], McMullenShephard [1968a]).

In this connection the following facts should be emphasized. An inspection of the proofs shows that most results on the upper-bound conjecture remain valid if one considers triangulations of the $(d-1)$ sphere $S^{d-1}$ instead of simplicial $d$-polytopes. The only exception concerns the case $v=d+3$, but even in this case the result remains valid due to a recent theorem of Mani [1970b]: Every triangulation of $S^{d-1}$ with $d+3$ vertices is isomorphic to the boundary complex of some simplicial $d$-polytope. However, since already for $d=4$ there exist triangulations of $S^{d-1}=S^{3}$ with only 8 vertices which are not isomorphic to the boundary complex of any 4-polytope (GrünbaumSreedharan [1967], Barnette [1970b], Barnette-Wegner [1970], Grünbaum [1970b]), one should distinguish between the upperbound conjecture for polytopes and the analogous but distinct one for triangulations of spheres. ${ }^{15}$ Similar distinctions should be made concerning the other problems discussed above. Though it is possible that the solutions of the two upper-bound problems coincide, the two classes certainly behave in distinct manners if one imposes the condition of central symmetry. For example, while a centrally symmetric 4-polytope with 12 vertices may have at most 46 facets (Grünbaum [1967a, §6.4], McMullen-Shephard [1968a]), there exist centrally symmetric triangulations of $S^{3}$ with 12 vertices and 48 facets (Grünbaum [1970b], [1970c], [1970d]).

For investigations related to the Kruskal-Katona Theorem 2.7 and dealing with cubical complexes see Harper [1964], Lindsey [1964], Bernstein [1967], Kruskal [1969], Clements-Lindström [1969], and Clements [1970]. ${ }^{16}$

${ }^{15}$ See Note 8 on page 1183.

16 See Note 12 on page 1184 . 
Besides the Euler characteristic $\chi(\mathbb{C})=\sum_{i \geq 0}(-i)^{i} f_{i}(\mathbb{C})$, various other "combinatorial invariants" of complexes have been investigated in algebraic topology. Among them are several integer-valued invariants, based on incidences of faces of various dimensions (see Bott [1952] and, in particular, Wu [1965], where additional references to the literature may be found). For many of them it seems that an investigation of their behavior for polytopes should be both interesting and feasible. For definiteness, we shall discuss only one such invariant, denoted $\chi_{2}^{+}$by $\mathrm{Wu}$ [1965]. Given a simplicial complex $\mathbb{C}$, let $\alpha_{i, j}(\mathcal{C})$ denote the number of ordered pairs $\left(F^{i}, F^{j}\right)$ consisting of an $i$-face $F^{i}$ and a $j$-face $F^{j}$ of $\mathfrak{e}$ such that $F^{i} \cap F^{j}=\varnothing$. Then $\chi_{2}^{+}$is defined as

$$
\chi_{z}^{+}(\mathbb{e})=\sum_{i, j \leq 0}(-1)^{i+j} \alpha_{i, j}(\mathbb{e}) .
$$

The statement " $\chi_{2}^{+}$is a combinatorial invariant" means that for every simplicial subdivision $\mathfrak{C}^{\prime}$ of the simplicial complex $\mathfrak{C}$ we have $\chi_{2}^{+}\left(\mathfrak{e}^{\prime}\right)=\chi_{2}^{+}(\mathfrak{e})$. (Concerning erroneous formulations of certain uniqueness statements of $\mathrm{Wu}$ [1965], Lee [1959] and other authors see Grünbaum [1969e].) Among problems concerning polytopes and $\chi_{2}^{+}$we mention:

(i) It is well known that $\chi_{2}^{+}(P)=\chi_{2}^{+}(B(P))=1-(-1)^{d}$ for every simplicial $d$-polytope $P$. Does the same relation hold for all $d$-polytopes? More generally, is $\chi_{2}^{+}$an invariant even in the sense that it remains unchanged for all cell-subdivisions of the given complex?

(ii) Is $\chi_{2}^{+}(P)=1-(-1)^{d}$ the only linear relation between the $\alpha_{i j}$ 's valid for all $d$-polytopes?

(iii) Do the $\alpha_{i j}$ 's of simplicial (or simple) $d$-polytopes satisfy equations analogous to the Dehn-Sommerville equations?

It is not hard to prove that the answer to both parts of (i) is affirmative, and it may be conjectured that the answers are affirmative in the other two cases as well. The situation is probably similar for other combinatorial invariants.

2.2. Angle-sums and related concepts. We now turn to a field which displays a fascinating interdependence between metric and combinatorial aspects of the theory of polytopes. Though its beginnings go back to Euclid or earlier, and contributions to it were made by people like Gram, Poincaré, Dehn, Sommerville and many others (see Grünbaum [1967a, Chapter 14] for an account of the history, and for references to the original papers; see also Carlitz [1961]), a 
deeper understanding was reached only during the last few years.

Let $P$ be a $d$-polytope in $d$-space, $F$ a $k$-face of $P$, and $z$ a point of the relative interior of $F$. The angle $\phi(F, P)$ of $P$ at $F$ is defined as the ratio of the content of $S \cap P$ to that of $S$, where $S$ is a sufficiently small $(d-1)$-sphere centered at $z$. It is easy to see that $\phi(F, P)$ is independent of $S$ and $z$, and that it coincides (up to a constant factor) with the usual "solid angle." We define, for $0 \leqq k \leqq d$, the $k$ th angle sum $\alpha_{k}(P)$ as the sum, over all the $k$-faces $F$ of $P$, of the corresponding angles $\phi(F, P)$. Hence, for example, $\alpha_{d}(P)=1$ and $\alpha_{d-1}(P)=\frac{1}{2} f_{d-1}(P)$. We define the $\alpha$-vector $\alpha(P)$ of $P$ by $\alpha(P)=\left(\alpha_{0}(P), \cdots, \alpha_{d-1}(P)\right)$.

As analogs to Euler's Theorem 2.2 and to the Dehn-Sommerville equations (Theorem 2.3) we have

THEOREM 2.14. The affine hull of the $\alpha$-vectors of all d-polytopes has dimension $d-1$. More precisely,

$$
\operatorname{aff}\left\{\alpha(P) \mid P \in \mathbb{P}^{d}\right\}=\left\{\left(\alpha_{0}, \cdots, \alpha_{d-1}\right) \mid \sum_{i=0}^{d-1}(-1)^{i} \alpha_{i}=(-1)^{d-1}\right\} .
$$

TheOREM 2.15. For each simplicial d-polytope $P$ and for each $k$ with $0 \leqq k \leqq d-1$ we have

$$
\sum_{i=k}^{d-1}(-1)^{i}\left(\begin{array}{l}
i+1 \\
k+1
\end{array}\right) \alpha_{i}(P)=(-1)^{d}\left(\alpha_{k}(P)-f_{k}(P)\right) .
$$

The older proofs of Theorems 2.14 and 2.15 were rather cumbersome (see Sommerville [1927], Höhn [1953], Grünbaum [1967a]). Recently, new approaches to those theorems and to many new results were developed (see below) and led to very simple proofs (Shephard [1967a], Perles-Shephard [1967b]).

In order to formulate an additional set of results we have to introduce some notation. First, we will use the symbol $\phi(F, P)$ even if $F$ is not a face of $P$, setting in that case $\phi(F, P)=0$. Next, denoting by $F_{1}^{d-1}, \cdots, F_{m}^{d-1}$ the $m=f_{d-1}(P)$ facets of $P$, we define, for a $k$-face $F$ of $P$,

$$
\delta(F, P)=1-\sum_{i=1}^{m} \phi\left(F, F_{i}^{d-1}\right) .
$$

We call $\delta(F, P)$ the angle deficiency of $P$ at $F$, and we define the $k$ th angle deficiency of $P$ by $\delta_{k}(P)=\sum_{F} \delta(F, P)$, the summation being over all the $k$-faces $F$ of $P$.

Clearly $\delta_{d-1}(P)=\delta_{d-2}(P)=0$ for each $P \in \mathcal{P}^{d}$.

Generalizing the well-known elementary result for $d=3$, Shephard [1968a] proved the following theorems (here and in other statements 
of this section, for sake of simplicity we formulate the results only for polytopes, or for simplicial polytopes, though more general results are known).

THEOREM 2.16. If $P$ is a d-polytope and $F$ a k-face of $P$, where $0 \leqq k \leqq d-3$, then $\delta(F, P)>0$.

THEOREM 2.17. If $P$ is a d-polytope then

$$
\sum_{i=0}^{d-3}(-1)^{i} \delta_{i}(P)=1-(-1)^{d} .
$$

Theorem 2.18. For every simplicial d-polytope $P$ and for each $k=0$, $1, \cdots, d-3$ we have

$$
\sum_{i=k}^{d-3}(-1)^{i}\left(\begin{array}{l}
i+1 \\
k+1
\end{array}\right) \delta_{i}(P)=(-1)^{d-1} \delta_{k}(P) .
$$

(Analogs of Theorems 2.15 and 2.18 for cubical polytopes have also been investigated.)

The main idea of the Perles-Shephard [1967b] and Shephard [1968a] approaches to angle-sums and deficiencies has been used in other contexts as well (see, for example, Fáry [1949], Banchoff [1967]). It belongs to integral geometry and utilizes connections between angles of polytopes and faces of suitable projections. Since integration over the Grassmannian manifold of all $j$-dimensional flats through a point (provided with the appropriate invariant measure) is used, attention may be restricted to $j$-flats which are in "general position" with respect to the faces of the polytope. With this approach, in which the angle-sums (or closely related entities) correspond to $j=1$, while deficiencies correspond to $j=2$, the results of Theorems 2.14 and 2.17 become, essentially, averaged forms of Euler's theorem for the various $(d-j)$-dimensional projections of the given polytope, while Theorems 2.15 and 2.18 are averaged versions of the Dehn-Sommerville equations. The method may be generalized to $j \geqq 3$, and yields a wealth of relations between "Grassmann angles" of polytopes (see Grünbaum [1968b]). Grassmann angles, of which the solid angles and the deficiencies are special cases, are additional entities giving information on the shape of a polytope near its various faces. The consideration of Grassmann angles helps to put into proper perspective the similarities and differences between the results for angle-sums and those for deficiencies. For $j=d-1$, the Grassmann angles are essentially identical with the exterior angles (see below, §2.3; compare also Banchoff [1967]). 
We return now to a discussion of angle-sums following PerlesShephard [1967b]; similar results hold for deficiencies (Shephard [1968a]) and, more generally, for Grassmann angles (Grünbaum $[1968 \mathrm{~b}])$. Let $P$ be a $d$-polytope in $d$-space, and let $L$ be a line not parallel to any face of $P$. We denote by $P(L)$ the $(d-1)$-polytope obtained by projecting $P$, parallel to $L$, onto a $(d-1)$-flat orthogonal to $L$; such a projection $P(L)$ of $P$ is called a regular projection. All the regular projections of $P$ belong to a finite number of different combinatorial types. Let $P_{1}, \cdots, P_{q}$ be $(d-1)$-polytopes representative of all the different combinatorial types of regular projections of $P$. With a slight abuse of notation, let $f\left(P_{i}\right)=\left(f_{0}\left(P_{i}\right), \cdots, f_{d-1}\left(P_{i}\right), 0\right)$. For a $d$-polytope $P$ let $a(P)$ denote the family of all $d$-polytopes affinely equivalent to $P$.

THEOREM 2.19. For every $d$-polytope $P$, $\operatorname{conv}\{\alpha(Q) \mid Q \in Q(P)\}=$ rel int $\operatorname{conv}\left\{\frac{1}{2}\left(f(P)-f\left(P_{i}\right)\right) \mid i=1, \cdots, q\right\}$.

Hence, in particular, $f(P)-2 \alpha(P)$ is a convex combination of the vectors $f\left(P_{i}\right), i=1, \cdots, q \cdot{ }^{17}$

We refrain from listing all the interesting results contained in the papers cited above; instead, following Perles-Shephard [1967a], we shall describe an application of those metric results to a combinatorial problem. The circle of ideas involved is the first attempt to treat in higher dimensions the type of problems we considered for 3-polytopes in $\$ 1.3$.

Let a $d$-polytope $P$ be called facet-forming provided there exists a $(d+1)$-polytope $Q$ such that each $d$-face of $Q$ is combinatorially equivalent to $P$. In case $d=2$ it is a simple consequence of Euler's equation that the only facet-forming 2-polytopes are the triangle, the quadrangle, and the pentagon. Easy examples (such as the duals of cyclic 4-polytopes) show that there exist facet-forming 3-polytopes with arbitrarily many 2 -faces (or vertices). The question whether there exist 3-or higher-dimensional non-facets (i.e., polytopes which are not facet-forming) was solved by Perles-Shephard. One of their criteria is derived from Theorem 2.19; using the notion of regular projection introduced above, it may be formulated as follows:

TheOREM 2.20. If $P$ is a $d$-polytope and if for some $j$ with $0 \leqq j \leqq d-2$ we have

$$
f_{j}(P) \geqq \frac{d+1-j}{d-1-j} \max \left\{f_{j}\left(P_{1}\right), \cdots, f_{j}\left(P_{q}\right)\right\}
$$

${ }^{17}$ See Note 13 on page 1184. 
then $P$ is a non-facet.

In case $d=3, j=1$, denoting by $h(P)$ the maximal possible number of edges belonging to a simple circuit in the graph of $P$, there follows:

TheOREM 2.21. If $P$ is a 3-polytope and if $h(P) \leqq \frac{1}{3} f_{1}(P)$, then $P$ is a non-facet.

The existence of 3-dimensional non-facets now follows by easy examples; simple 3-dimensional non-facets may be obtained using Theorem 1.25. Many additional types of non-facets of dimensions $\geqq 3$ were also obtained by Perles-Shephard [1967a] through different applications of Theorem 2.20. We quote only one of their results, recalling that a $d$-polytope $P$ is neighbourly provided every $\left[\frac{1}{2} d\right]$ of its vertices determine a face of $P$ (see Grünbaum [1967a] or McMullenShephard [1968b] for a more detailed account; the cyclic polytopes are neighbourly, but there are neighbourly polytopes not combinatorially equivalent to cyclic polytopes).

Theorem 2.22. For $d \neq 3$, there exists a function $v(d)$ such that each simplicial, neighbourly d-polytope $P$ satisfying $f_{0}(P)>v(d)$ is a nonfacet.

The determination of the smallest possible $v(d)$ seems to be a difficult problem; in the Perles-Shephard treatment it is made to depend on the upper-bound conjecture (see above, \$2.1). The estimate $v(d) \leqq\left[\frac{1}{2} d\right]^{2}-2$ for $d \geqq 4$ (along with better estimates) is given by Perles-Shephard [1967a]. The existence of $v(3)$ is still undecided.

Using the case $d=4, j=2$, of Theorem 2.20, Barnette [1969b] has recently established the existence of simple 4-polytopes which are non-facets. One of Barnette's tools, interesting also for its own sake, is an analog of Theorem 1.25, dealing with 2-manifolds contained in the 2-skeletons of simple 4-polytopes.

The existence of simple non-facets of dimensions $\geqq 5$ is likely, but still undecided. Many other open problems on non-facets are mentioned by Perles-Shephard [1967a]; possibly the most provocative is: Are all simplicial $d$-polytopes with $d+4$ or more vertices nonfacets?

With only minor modifications, the results on angle-sums, deficiencies and Grassmann angles mentioned above may be reformulated for polyhedral cones, or for spherical polytopes (see Perles-Shephard [1967b], Shephard [1968a], Grünbaum [1968b]). Probably more important, however, are extensions of the notions and results to var- 
ious classes of polyhedra or complexes embedded in Euclidean spaces; some of the results that have been obtained are polyhedral analogues of well-known differential-geometric facts. It would lead us too far to attempt to reproduce here the relevant material. For different generalizations of Grassmann angles (or their special cases) to polyhedra the reader is referred to Allendoerfer-Weil [1943], Banchoff [1967], Hadwiger [1969b], Mani [1970c], and Larman-Mani [1970b]. In many respects it seems that the investigation of the whole field has barely begun, and that important developments and applications should be expected.

One of the most important aspects of the angle-sums and other entities discussed in the present section is that they are associated specifically with geometric complexes. Therefore they are one of the few tools (see also below, Chapter 3 ) that enable us to establish results for geometric complexes which are not necessarily valid for abstract or topological complexes.

For example, in contrast to Theorems 2.20 and 2.21, it is not known whether there exists a 3-polytope $P$ such that no cell complex homeomorphic to the 3-sphere $S^{3}$ has all 3-cells isomorphic to $P$.

2.3. Steiner points, mean widths, mixed volumes. Steiner points and the other functions discussed below provide excellent examples of the interplay between differential-geometric and discrete approaches to a topic. Though the recent development of the subject was rather rapid, many problems are still open; we are probably even lacking the proper point of view of the whole area.

Following Steiner's [1840] introduction in 1840 of a point $s(C)$ as the curvature-centroid of a planar curve $C$, different authors have extended the definition of the Steiner point $s(C)$ to higher-dimensional compact convex sets $C$, dispensing at the same time with differentiability assumptions (see Grünbaum [1967a, p. 313] for an account of the history, and for references to the literature). A useful definition of the Steiner point $s(C)$ of a compact convex set $C$ in $E^{d}$ is

$$
s(C)=\int_{S^{d-1}} u h(u, C) d \mu,
$$

where $\mu$ is the normalized measure on the unit $(d-1)$-sphere, and $h(u, C)$ is the value of the supporting function of $C$ in the direction of the unit vector $u$. For other formulas for $s(C)$ see Shephard [1966a] and Flanders [1966].

Among the most important properties of Steiner points are:

(a) Additivity. $s\left(C_{1}\right)+s\left(C_{2}\right)=s\left(C_{1}+C_{2}\right)$ for all compact convex sets $C_{1}, C_{2}$, the addition on the right being vector addition of sets. 
(b) Invariance. $s(T C)=T s(C)$ for all compact convex sets $C$ and all orthogonal transformations $T$.

(c) Continuity. $s(C)$ is a continuous function of $C$, the space of all compact convex sets in $E^{d}$ being metrized by the Hausdorff distance.

As a solution of a problem raised in Grünbaum [1963b] we have:

THEOREM 2.23. The Steiner point $s(C)$ is the only vector-valued function defined for all compact convex sets $C$ in $E^{d}$ which satisfies (a), (b), and (c).

The history of Theorem 2.23 is rather interesting. For $d=2$ it was proved by Shephard [1968b]. Schmitt [1968] published a proof for the general case which, however, was incomplete at a crucial step. Berg [1969b] proved the theorem for $d=3$, and Meyer [1969] proved it for all $d$ assuming uniform continuity in (c). Theorem 2.23 as formulated here was finally established by Schneider [1970]. Sallee [1970] showed that Theorem 2.23 fails if the continuity assumption (c) is dropped.

Some of the interest in Theorem 2.23 (and in the results discussed below) derives from the fact that Steiner points are useful in various seemingly unrelated investigations (see, for example, Firey-Grünbaum [1964], Flanders [1966], Shephard [1964b], Berg [1969a]). On the other hand, a crucial part in the proofs of Theorem 2.23 is played by certain spaces of "asymmetry classes" of convex sets, which are interesting for their own sake and seem to be applicable to different other problems as well (see Ewald [1965], Ewald-Shephard [1966], Schmitt [1967], Shephard [1966b]; for related questions see also Bantegnie [1965], Shephard-Webster [1965]).

The Steiner point $s(P)$ of a $d$-polytope $P$ may alternatively be defined as

$$
s(P)=\sum_{i=1}^{f_{0}(P)} V_{i} \psi\left(V_{i}, P\right),
$$

where $\psi(V, P)$ is the exterior angle of $P$ at its vertex $V$ (that is, the (normalized) measure of the set of those unit vectors $u$ which are outer normals of hyperplanes supporting $P$ at $V$ ). In order to shorten the exposition of the remaining parts of the present section, we make the following notational agreement. If $g$ is a function defined for all polytopes $P$, we put $g_{i}(P)=\sum_{j} g\left(F_{j}^{i}\right)$, the summation being over all the $i$-faces $F_{j}^{i}$ of $P$. Thus, for example, $g_{d}(P)=g(P)$ for each $d$-polytope $P$.

Using this notation, the rather surprising results of Shephard [1966a] (see also Grünbaum [1967a, \$14.3]) may be presented in 
the following form, analogous to the equations of Euler and of DehnSommerville.

THEOREM 2.24. If $P$ is a d-polytope then

$$
\sum_{i=0}^{d}(-1)^{i} s_{i}(P)=(-1)^{d} s_{d}(P) .
$$

Theorem 2.25. If $P$ is a simple $d$-polytope and if $0 \leqq k \leqq d$, then

$$
\sum_{i=0}^{k}(-1)^{i}\left(\begin{array}{l}
d-i \\
d-k
\end{array}\right) s_{i}(P)=s_{k}(P) .
$$

This system is easily seen to contain $\left[\frac{1}{2}(d+1)\right]$ linearly independent equations, and it seems likely that every other linear relation between the $s_{i}(P)$ 's, valid for all simple $d$-polytopes $P$, is linearly dependent on them. This problem is still open, as is the more general problem of characterizing the $(d+1)$-tuples $\left(s_{0}(P), \cdots, s_{d}(P)\right)$ which correspond to simple $d$-polytopes $P$. Also open are the analogous questions concerning Theorem 2.24.

Another open problem is whether each vector-valued function $s(P)$ defined and continuous (in the Hausdorff metric) only for polytopes, which satisfies (a) and (b) for polytopes, is necessarily the Steiner point of $P$.

Many other functions defined for polytopes exhibit a behavior similar to that of Steiner points. As one example, which is both of independent interest and useful in proofs of other relations, we mention the following result of Shephard [1968c], in which $h(u, P)$ is the supporting function of $P$ (see above for the convention regarding the definition of $h_{i}$ ).

Theorem 2.26. For every $d$-polytope $P$ and for each vector $u$,

$$
\sum_{i=0}^{d}(-1)^{i} h_{i}(u, P)=-h(u,-P) .
$$

The remarkable feature in this result is the appearance of $-P$. While the negative sign here could be transferred to $u$, it seems to be rather naturally attached to $P$ in view of the next result. For any polytope $P$, and integer $r, 1 \leqq r \leqq d$, and any convex bodies $K_{r+1}, \cdots$, $K_{d}$, let

$$
v^{(r)}\left(P ; K_{r+1}, \cdots, K_{d}\right)=v\left(P, \cdots, P, K_{r+1}, \cdots, K_{d}\right)
$$

be the mixed volume of the bodies $P$ ( $r$ times), $K_{r+1}, \cdots, K_{d}$. Then we have (Shephard [1968c]): 
TheOREM 2.27. For every $d$-polytope $P$ and any convex bodies $K_{r+1}, \cdots, K_{d}$,

$$
\sum_{i=r}^{d}(-1)^{i} v_{i}^{(r)}\left(P ; K_{r+1}, \cdots, K_{d}\right)=(-1)^{r} v^{(r)}\left(-P ; K_{r+1}, \cdots, K_{d}\right) .
$$

As a special case of Theorem 2.27 (if $r=1$ and $K_{2}=K_{3}=\ldots=K_{d}$ is the unit ball of $E^{d}$ ) we have the following theorem of Shephard [1968e]:

THEOREM 2.28. If $m(P)$ denotes the mean width of the polytope $P$, then for every $d$-polytope $P$ we have

$$
\sum_{i=1}^{d}(-1)^{i+1} m_{i}(P)=m(P) .
$$

In analogy to the situation concerning Steiner points, the functions $m_{i}$ of simple or quasi-simple $d$-polytopes satisfy additional linear equations, which are derivable from the Dehn-Sommerville equations (Shephard [1968e]).

Though we do not know of any causal relationship, it seems worthwhile to recall here a result similar in form to Theorem 2.27, and to the Dehn-Sommerville equations for simple $d$-polytopes. For a compact convex set $P \subset E^{d}$ let $v^{(r)}(P)=v^{(r)}\left(P ; K_{r+1}, \cdots, K_{d}\right)$, where $K_{r+1}=\cdots=K_{d}$ is the unit ball of $E^{d}$. Then we have

Theorem 2.29. If $K$ is a convex body of constant width 1 in $E^{d}$, then

$$
\sum_{i=r}^{d}(-1)^{r}\left(\begin{array}{l}
d-r \\
d-i
\end{array}\right) v^{(i)}(K)=(-1)^{d_{v}(r)}(K)
$$

for $r=0,1, \cdots, d$.

For $d=3$ this result is due to Blaschke [1915]; proofs of the general case were given by Dinghas [1940] and Santaló [1946]; an especially simple proof is due to Debrunner [1955]. It would be very interesting to investigate the possibility of a deeper connection between Theorem 2.29 and the formally similar results discussed in the other parts of this paper.

Another aspect of Steiner points deserves special mention. Let $g(C)$ be any function defined for all sets that appear as its arguments; let a family $\mathfrak{C}=\left\{C_{1}, \cdots, C_{n}\right\}$ of sets be given, and let $1 \leqq r \leqq n$. We define 


$$
g^{(r)}(\mathcal{C})=\sum_{\left(i_{1}, \cdots, i_{r}\right)} g\left(C_{i_{1}} \cap \cdots \cap C_{i_{r}}\right)
$$

the summation being over all $r$-tuples $\left(i_{1}, \cdots, i_{r}\right)$ such that $1 \leqq i_{1}$ $<i_{2}<\cdots<i_{r} \leqq n$. Then we have (Sallee [1966]):

TheOREM 2.30. Let $\mathfrak{K}=\left\{K_{1}, \cdots, K_{n}\right\}$ be a family of compact convex sets in $E^{d}$ such that $K=K_{1} \cup \ldots \cup K_{n}$ is convex. Then

$$
s(K)=\sum_{i=1}^{n}(-1)^{i+1} s^{(i)}(\mathcal{K}) .
$$

In other words, the function $s$ has a restricted kind of the valuation property. This restriction is related to the fact that $s(C)$ was defined only for convex $D$. Theorem 2.30 suggests an extension of the definition of $s$ to the class of sets in $E^{d}$ which are unions of finitely many bounded convex sets. Investigations in this and related directions were carried out by Hadwiger [1969a], [1970], Sallee [1966], [1968], Mani [1970c], and Perles-Sallee [1970]. One byproduct of the results obtained by these authors is a clarification of the quite surprising interrelations between the properties of a function to be valuationlike, and to satisfy Euler-type relations; the results are relevant to different quantities discussed in the present chapter. For more precise statements the reader is referred to the original papers. As pointed out by W. J. Firey (private communication), some of those results could also be obtained from the more classically-oriented work of Hadwiger [1957, Chapter 6] on functionals defined on the space of sets which are unions of finitely many convex sets. For other results in this direction see Volland [1957], Fáry [1960], [1961], Müller [1967] and Scherk [1969].

3. Gale transforms and Gale diagrams. Certain algebraic constructions, traces of which may be found in some works of T. S. Motzkin and D. Gale, were systematically developed by M. A. Perles and used to establish a number of results on polytopes which were completely unreachable by other methods. Perles' results were published in Grünbaum [1967a], and formed the starting point for recent investigations by McMullen, Shephard, and others. The aim of the present chapter is to give a summary of some of those results and of material related to the theorems obtained in this manner. For a detailed exposition of the Gale diagram technique see McMullenShephard [1970c], for a brief introduction see Stoer-Witzgall [1970].

3.1. Definitions and techniques. Let $X=\left(x_{1}, \cdots, x_{n}\right)$ be a sequence of (not necessarily distinct) points $x_{1}, \cdots, x_{n}$ in Euclidean $d$-space 
$E^{d}$. For sake of simplicity we shall assume that aff $X=E^{d}$, though the theory may be developed without this restriction. A sequence $\bar{X}=\left(\bar{x}_{1}, \cdots, \bar{x}_{n}\right)$ is said to be $a$ Gale transform of $X$ provided the matrix

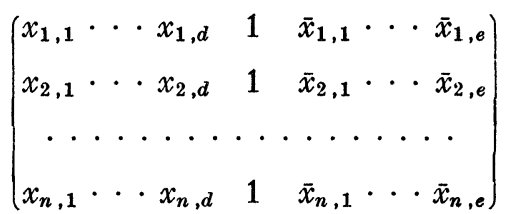

is non-singular and provided its last $e=n-d-1$ columns are orthogonal to its first $d+1$ columns; here $x_{i}=\left(x_{i, 1}, \cdots, x_{i, d}\right)$ and $\bar{x}_{j}=\left(\bar{x}_{j, 1}, \cdots, \bar{x}_{j, e}\right)$.

Simple dimensionality arguments establish the existence of $\bar{X} \subset E^{e}$ for every $X$ with aff $X=E^{d}$. Also, it is clear that $\sum_{i=1}^{n} \bar{x}_{i}=0$, that $\bar{X}$ positively spans $E^{e}$, and that $\bar{X}$ is also a Gale transform of $X+y$ $=\left(x_{1}+y, \cdots, x_{n}+y\right)$ for each $y \in E^{d}$. Hence it is possible and sometimes convenient to replace $X$ by a translate $X^{\prime}$ such that $\sum_{i=1}^{n} x_{i}^{\prime}=0$. If $X$ itself satisfies this relation, there is a duality between $X$ and $\bar{X}$, since $X$ is in this case a Gale transform of $\bar{X}$. This duality has a particularly important consequence (which may also be established directly):

If $Z=\left(z_{1}, \cdots, z_{n}\right)$ is an $n$-tuple which spans $E^{n-d-1}$, such that $\sum_{i=1}^{n} z_{i}=0$, then there exists $X \subset E^{d}$ such that $Z$ is a Gale transform of $X$.

It is not hard to show that if $\bar{X}$ and $\bar{X}^{\prime}$ are Gale transforms of the same $X$ then $\bar{X}$ and $\bar{X}^{\prime}$ are linearly equivalent. Similarly, it is easy to follow the influence of various other types of transformations (affine, projective) on Gale transforms.

The relevance of Gale transforms for the theory of polytopes is due to the following fundamental facts.

TheOREM 3.1. Let $X$ be the set of vertices of a d-polytope $P \subset E^{d}$, let $X=X_{1} \cup X_{2}$ where $X_{1} \neq \varnothing \neq X_{2}$ and $X_{1} \cap X_{2}=\varnothing$, and let $\bar{X}_{2}$ be the subset of $\bar{X}$ consisting of the points which correspond to the points of $X_{2}$. Then conv $X_{1}$ is a proper face of $P$ if and only if $0 \in$ rel int conv $\bar{X}_{2}$.

In other words, $X_{1}$ is the set of all vertices of some face of $P$ is and only if $\bar{X}_{2}$ positively spans its linear hull.

Theorem 3.2. An n-tuple $X=\left(x_{1}, \cdots, x_{n}\right)$ which spans $E^{d}$ is the set of vertices of some $d$-polytope $P$ if and only if

either $\bar{x}_{i}=0$ for $i=1, \cdots, n$ (in which case $P$ is a $d$-simplex); 
or else for every open halfspace $H$ of $E^{n-d-1}$ such that $0 \in \mathrm{bd} H$, we have $\operatorname{card}\left\{i \mid \bar{x}_{i} \in H\right\} \geqq 2$.

Hence Gale transforms permit the translation of questions regarding the facial structure of polytopes to questions on positive dependences, and vice versa. Paralleling Theorems 3.1 and 3.2 are criteria for $P$ to be simplicial, or to be a pyramid, etc., expressed as simple properties of $\bar{X}$. This is particularly advantageous for the theory of polytopes in cases $n-d$ is small, since then the question becomes one concerning positive dependence in low-dimensional spaces, which are sometimes easier to handle. In the following sections we shall discuss in detail a number of such applications. For another application see Reay [1968b].

If one wishes to use Gale transforms of $X=\operatorname{vert} P$ in order to study the facial structure of the polytope $P$, it follows from Theorem 3.1 that $\bar{X}$ may be submitted to various changes which will not affect its usefulness for the investigation of the structure of $P$. For example, replacing each non-zero $\bar{x}_{i}$ by $\hat{x}_{i}=\bar{x}_{i} /\left|\bar{x}_{i}\right|$ leads to a sequence $\hat{x}=\left(\hat{x}_{1}, \cdots, \hat{x}_{n}\right)$ which is not necessarily a Gale transform of $X$ (or of any other sequence); but the criterion of Theorem 3.1 still relates the facial structure of $X$ with the positive-dependence properties of $\hat{X}$. Such sequences $\hat{X}$ are called Gale diagrams of $X$; as we shall see in $\S 3.2$, the consideration of Gale diagrams is sometimes even more useful than that of Gale transforms.

The above facts and terminology are taken from Grünbaum [1967a]; in the papers of McMullen and Shephard which will be discussed below (as well as in Grünbaum-Shephard [1969]) a slightly different point of view and terminology are used.

One of the less attractive aspects of the algebraic treatment of Gale transforms described above is the apparent arbitrariness of the whole concept, and the lack of intuitive meaning. This is alleviated by the following observation which may be used as a starting point for the theory of Gale transforms (McMullen [1968a]):

Theorem 3.3. Let $E^{d}$ and $E^{n-d-1}$ be orthogonal subspaces through the origin 0 of $E^{n-1}$, and let $T^{n-1}$ be a regular $(n-1)$-simplex with centroid 0 . If $X$ is the orthogonal projection of vert $T^{n-1}$ into $E^{d}$, and if $\bar{X}$ is the orthogonal projection of vert $T^{n-1}$ into $E^{n-d-1}$ then $X$ is a Gale transform of $X$, and $X$ is a Gale transform of $\bar{X}$.

For another geometric interpretation of Gale transforms see McMullen [1968a], [1968b], McMullen-Shephard [1968a].

Guided by these geometric interpretations of Gale transforms, McMullen-Shephard [1968a], [1970b] were also able to devise 
analogous methods for the treatment of centrally symmetric polytopes, or polytopes with a higher-dimensional axis of symmetry. We shall briefly describe the derivation of the centrally symmetric case from the Gale transforms introduced above.

Let $X=\left\{x_{1},-x_{1}, x_{2}, \cdots, x_{n},-x_{n}\right\}$ be a centrally symmetric set of points in $E^{d}$ which linearly spans $E^{d}$. Among the Gale transforms $\bar{X}$ of $X$ there are some having the form

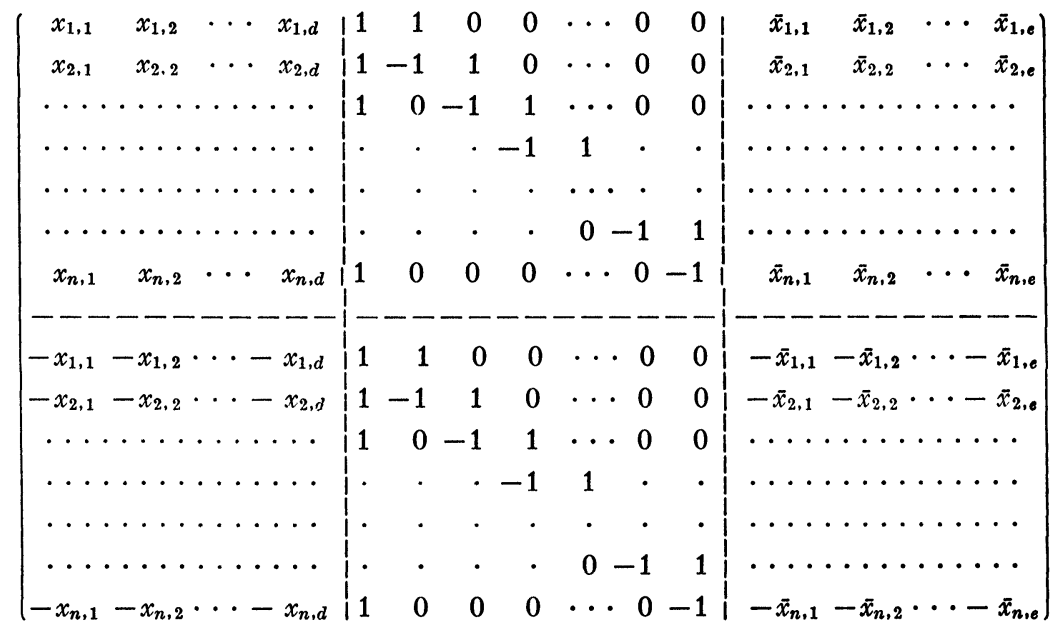

where the last $e=n-d$ columns are chosen orthogonal to the first $d$ columns, all of them being automatically orthogonal to the central $n$ columns. In order to determine the facial structure of $X$ it is not necessary to specify explicitly all of $\bar{X}$; the knowledge that we are dealing with a centrally symmetric set, together with the $n$-tuple $\tilde{X}=\left(\tilde{x}_{1}, \cdots, \tilde{x}_{n}\right)$, where $\tilde{x}_{i}=\left(\bar{x}_{i, 1}, \cdots, \bar{x}_{i, e}\right) \subset E^{e}$, determine $\bar{X}$. $\tilde{X}$ is called a centrally symmetric transform of $X$. Using the above definition, Theorem 3.1 may easily be translated into the following result, first obtained by a different method by McMullen [1968a] and McMullen-Shephard [1968a].

TheOREM 3.4. Let $X=\left\{x_{1},-x_{1}, \cdots, x_{n},-x_{n}\right\}$ be the vertices of $a$ $d$-polylope $P \subset E^{d}$ centered at 0 , and let each $\epsilon_{i}$ be +1 or -1 . Then conv $\left\{\epsilon_{1} x_{i_{1}}, \cdots, \epsilon_{r} x_{i_{r}}\right\}$ is a face of $P$ if and only if

$$
\epsilon_{1} \tilde{x}_{i_{1}}+\cdots+\epsilon_{r} \tilde{x}_{i_{r}} \in \text { rel int conv } Y \text {, }
$$

where $Y$ is the set of the $2^{n-r}$ points of the form

$$
\pm \tilde{x}_{j_{1}} \pm \tilde{x}_{j_{2}} \pm \cdots \pm \tilde{x}_{j_{n-r}}
$$

with $\left\{j_{1}, \cdots, j_{n-r}\right\}=\{1, \cdots, n\} \backslash\left\{i_{1}, \cdots, i_{r}\right\}$. 
In the investigation of centrally symmetric polytopes the advantage of $\tilde{X}$ over $\vec{X}$ (or $\hat{X}$ ) is in the presence of fewer points ( $n$ instead of $2 n)$ in a space of lower dimension $(n-d$ instead of $2 n-d-1)$. A disadvantage is the form of the condition of Theorem 3.4, which is much harder to use than the condition of Theorem 3.1.

For a discussion of Gale transforms appropriate for polytopes having a higher-dimensional axis of symmetry see McMullen-Shephard [1970a] and McMullen [1968b].

Before turning to a somewhat detailed description of some of the achievements of Gale transforms techniques in the general theory of polytopes, we shall conclude the present section by a brief discussion of two recent variants of the technique. ${ }^{18}$ One of them is particularly adapted to the study of zonotopes and of arrangements of hyperplanes, the other to the investigation of positive bases.

Zonotopes are polytopes which may be represented as vector sums of segments. Though they form a rather specialized subclass of the centrally symmetric polytopes, they have important connections with various areas of analysis (in particular, vector measures) and geometry. Bolker [1969] is an excellent survey of known results, and should be consulted for references to the voluminous literature. See also Bolker [1970], Shephard [1967b], Schneider [1967a], Lindquist [1968], and McMullen [1970f] for some related results. A major advance in the theory of zonotopes was recently achieved by McMullen [1970e]. The starting point of McMullen's work is the observation that if a $d$-zonotope $Z$ is the vector sum of segments $S_{i}, i=1, \cdots, k$, (where no generality is lost by assuming $S_{i}=-S_{i}=\operatorname{conv}\left\{v_{i},-v_{i}\right\}$ ) then the facial structure of $Z$ may be related to the centrally symmetric transforms $\bar{V}$ of the set $\left\{ \pm v_{1}, \cdots, \pm v_{k}\right\}$. Among the most striking results of McMullen [1970e] on zonotopes we mention only:

(i) For $d \geqq 2$ and $n \geqq d+2$ there exists a one-to-one correspondence between the isomorphism types of cubical $d$-zonotopes with $n$ zones and cubical $(n-d)$-zonotopes with $n$ zones.

(ii) An enumeration of the isomorphism types of cubical, and of all, $d$-zonotopes with $n$ zones, for $n \leqq d+2$.

We recall here the notion of a d-arrangement. By this is meant a finite set of $(d-1)$-hyperplanes in the real projective $d$-space, such that no point belongs to all the hyperplanes. The $d$-arrangements

18 See Note 14 on page 1184. 
were the subject of many investigations; the topic acquired additional interest recently due to its connection with pattern recognition and similar problems. There are many connections between arrangements and polytopes (see Grünbaum [1967a, Chapter 18] for an account of some of the results, and for references). More recent material may be found in Grünbaum [1970d], [1970e], Bonnice-Kelly [1970], Rottenberg [1970], Alexanderson-Wetzel [1970], and Canham [1969].

Due to the one-to-one correspondence between $(d+1)$-zonotopes and $d$-arrangements (see, for example, Coxeter [1962]), the above results on zonotopes led McMullen [1970e] to an enumeration of the different isomorphism types of $d$-arrangements with at most $d+3$ hyperplanes, to the fact that for $n=d+1, d+2$, or $d+3$, there is only one type of simple $d$-arrangements, and-most surprisingly - to the following reformulation of (i):

THEOREM 3.5. If $d \geqq 1$ and $n \geqq d+3$ there is a one-to-one correspondence between the isomorphism types of simple $d$-arrangements of $n$ hyperplanes and simple $(n-d-2)$-arrangemenis of $n$ hyperplanes.

Theorem 3.1 may clearly be used "in reverse" to obtain information on positive dependence properties of $\bar{X}$ from the facial structure properties of $X$. However, if positive dependence is the main object of investigation it is more advantageous to use a slightly different form of the Gale transform technique by not allotting any particular role to the column vector having all components equal to 1 . This approach was developed in a most effective fashion by Shephard [1970a ] and applied by him to the study of positive bases. Along with interesting new results, Shephard [1970a] obtained simplified proofs of some results established previously by Davis [1954], McKinney [1962], Bonnice-Klee [1963], and Reay [1965a], [1965b], [1966], [1967]. Some of the ideas concerning positive bases go back to Steinitz [1913] and Minkowski [1897], [1903] and are in many ways relevant to the theory of polytopes. Steinitz's work is related to the theorems of Carathéodory and Helly on convex hulls, and on intersections of convex sets; see Danzer-Grünbaum-Klee [1963] for a survey of known results and for references, Bonnice-Reay [1969] and Hansen-Klee [1969] for some recent developments. Minkowski's work on the determination of polytopes by their "area functions" leads to results (see Firey-Grünbaum [1964])which may be viewed as refinements of some theorems concerning positive bases, and to "primitive polytopes" (compare Steinitz [1909], Fejes Tóth [1962], 


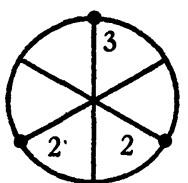

$(7,19,24,12)$

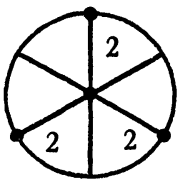

$(7,18,20,9)$

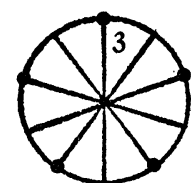

$(7,18,22,11)$

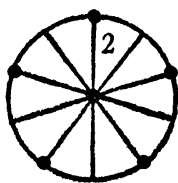

$(7,18,20,9)$

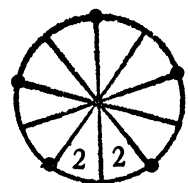

$(7,19,24,12)$

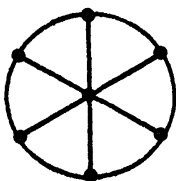

$(7,15,14,6)$

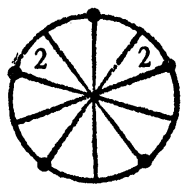

$(7,20,26,13)$

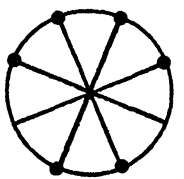

$(7,16,16,7)$

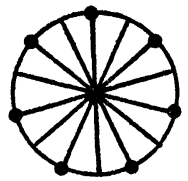

$(7,21,28,14)$

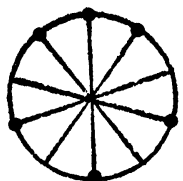

$(7,17,18,8)$
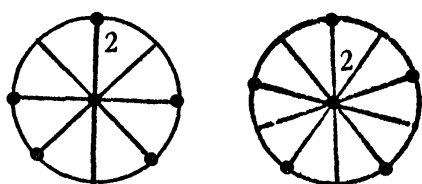

$(7,17,18,8)$
$(7,16,16,7)$
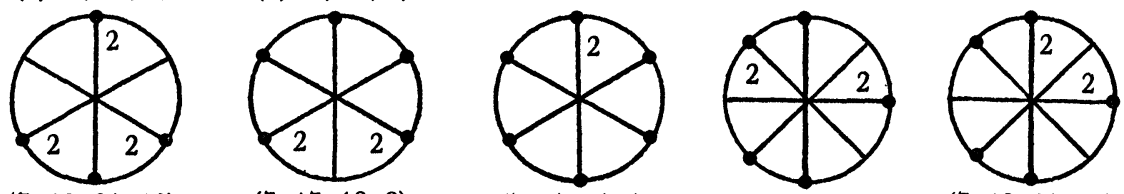

$(7,18,21,10)$

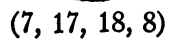

$(7,17,17,7)$

$(7,19,23,11)$

$(7,18,21,10)$
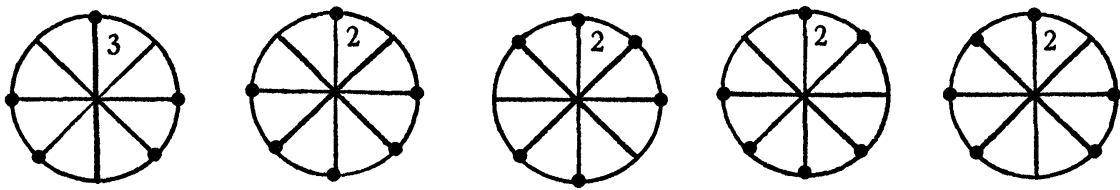

$(7,20,24,11)$

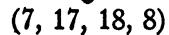

$(7,18,20,9)$

$(7,18,20,9)$
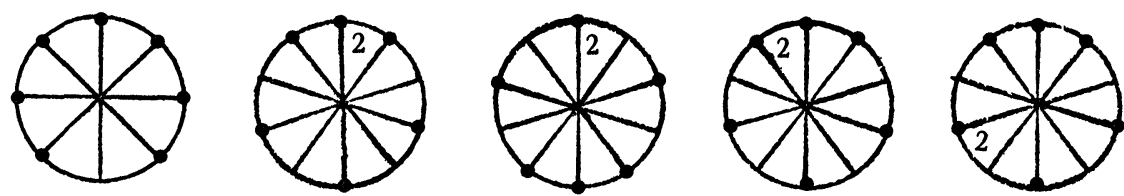

$(7,18,19,8)$

$(7,17,19,9)$

$(7,19,23,11)$

$(7,18,21,10)$
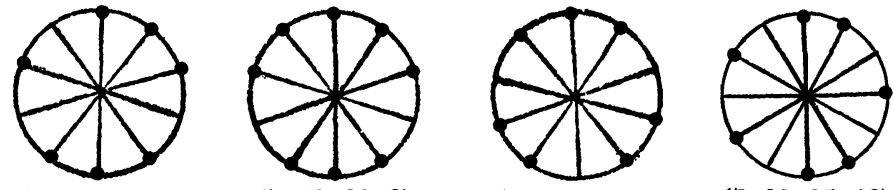

$(7,19,22,10)$

$(7,19,22,10)$

$(7,20,25,12)$

$(7,19,23,11)$

Figure 5 
Schneider [1967b]). Related is also the problem of "fixing sets" for polytopes (see Grünbaum [1964], Bollobás [1967], Mani [1970d]). For details the reader should consult the papers mentioned.

3.2. Enumeration of isomorphism types. Problems of enumeration of different isomorphism types of polytopes were among the first aims of the study of polytopes (see, for example, Euler [1752a], [1752b], Steiner [1830], Kirkman [1854], Cayley [1862]). Though much work was done in recent years concerning the enumeration of 3polytopes or of special kinds of 3-polytopes (see, among others, Grace [1965], Bowen-Fisk [1967], Federico [1969], Jucovič [1962], Rademacher [1965], Guy [1967], Brown [1965], Tutte [1968]), only few advances have been made concerning higher-dimensional polytopes. The following brief historical survey is believed to be complete. We shall use $c(v, d)$ to denote the number of different isomorphism types of $d$-polytopes with $v$ vertices, $c_{s}(v, d)$ for the number of simplicial ones among them.

The first results on the enumeration of higher-dimensional polytopes were obtained by Victor Schlegel and by Max Brückner. In 1891 Schlegel [1891] proved $c_{s}(d+2, d)=\left[\frac{1}{2} d\right]$, and in 1893 Brückner [1893] listed the simplicial 4-polytopes with 6 and 7 vertices, as well as all 4-polytopes with 6 vertices; he also attempted to enumerate all 4-polytopes with 7 vertices. Regarding $c(v, d)$ for $d \geqq 4$ no new results seem to have been obtained till 1967. In Grünbaum [1967a] it was shown that $c(d+2, d)=\left[\frac{1}{2} d^{2}\right]$, and the $d$-polytopes with $d+3$ vertices were enumerated for $d \leqq 6$. Lloyd [1970] found explicit formulae for $c(d+3, d)$ for all $d$. These recent results, as well as the result of Perles mentioned below, were obtained using Gale diagrams.

Concerning simplicial $d$-polytopes Schoute [1905] rediscovered the value of $c_{s}(d+2, d)$, and Brückner [1909] attempted to find $c_{s}(8,4)$. Due in part to difficulties rooted in the difference between the notion of boundary complex of a polytope, and that of triangulation of a sphere (see below, §3.4), Brückner's attempt failed and the enumeration of simplicial 4 -polytopes with 8 vertices was accomplished only in Grünbaum-Sreedharan [1967]. The values of $c_{s}(d+3, d)$ were determined by M. A. Perles for all $d$ (see Grünbaum [1967a]).

In Tables 1 and 2 we have collected all the available information on $c(v, d)$ and $c_{s}(v, d)$. In Figure 5 we represent (by their contracted standard Gale diagrams, see below) all 4-polytopes with 7 vertices, together with the corresponding $f$-vectors.

We have already mentioned in $\$ 3.1$ that the facial structure of a polytope $P$ with vertices $V$ is determined by the Gale transform $\bar{V}$ of $V$. More precisely, the isomorphism type of $P$ depends on the family 


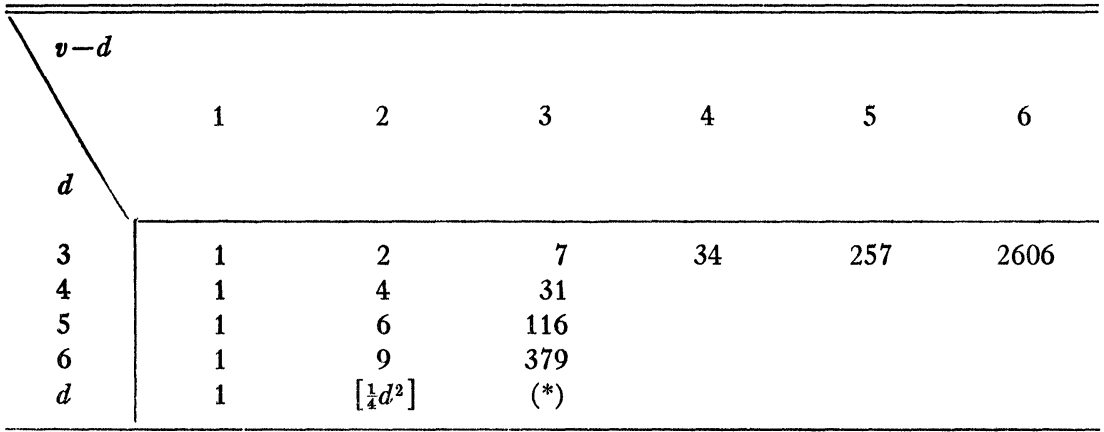

TABLE 1. The numbers $c(v, d)$ of different isomorphism types of $d$-polytopes with $v$ vertices. The values for $d=3$ are due to Hermes [1899] (for $v \leqq 8$ ) and Federico [1969] (for $v \leqq 9$ ), those for $v=d+3$ are due to M. A. Perles and have been independently checked by the author (see Grünbaum [1967a; $\$ 6.3]$ ). $\left(^{*}\right)$ was determined by Lloyd [1970].

\begin{tabular}{|c|c|c|c|c|c|c|c|c|c|}
\hline \multicolumn{10}{|c|}{$v-d$} \\
\hline 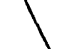 & 1 & 2 & 3 & 4 & 5 & 6 & 7 & 8 & 9 \\
\hline 3 & 1 & 1 & 2 & 5 & 14 & 50 & 233 & $1249^{*}$ & $7595^{*}$ \\
\hline 4 & 1 & 2 & 5 & $37 * *$ & & & & & \\
\hline 5 & 1 & 2 & 8 & & & & & & \\
\hline 6 & 1 & 3 & 18 & & & & & & \\
\hline 7 & 1 & 3 & 29 & & & & & & \\
\hline 8 & 1 & 4 & 57 & & & & & & \\
\hline 9 & 1 & 4 & 96 & & & & & & \\
\hline 10 & 1 & 5 & 183 & & & & & & \\
\hline 11 & 1 & 5 & 318 & & & & & & \\
\hline 12 & 1 & 6 & 603 & & & & & & \\
\hline 13 & 1 & 6 & 1080 & & & & & & \\
\hline 14 & 1 & 7 & 2047 & & & & & & \\
\hline 15 & 1 & 7 & 3762 & & & & & & \\
\hline$d$ & 1 & $\left\lceil\frac{1}{2} d\right]$ & $(* * *)$ & & & & & & \\
\hline
\end{tabular}

TABLE 2. The numbers $c_{s}(v, d)$ of different isomorphism types of simplicial $d$-polytopes with $v$ vertices. The values $*$ are due to Bowen-Fisk [1967], ${ }^{* *}$ is from Grünbaum-Sreedharan [1967], while the value $(* * *)$ as determined by M. A. Perles is reproduced in Grünbaum [1967a, p. 113].

of subsets $\bar{W}$ of $\bar{V}$ having the property

$$
0 \in \operatorname{rel} \text { int conv } \bar{W} \text {. }
$$

Therefore, if a set $\bar{X}$ is in a one-to-one correspondence with $\bar{V}$ and 
has the property that $\left(^{*}\right)$ holds if and only if the corresponding subset of $\bar{X}$ satisfies the analogous relation, the set $\bar{X}$ may be used instead of $\bar{V}$ for the determination of the facial structure of $P$. Any such set $\bar{X}$ is called a Gale diagram of $V$ or of $P$. If each point of $\bar{X}$ is either the origin, or else is on the unit sphere, $\bar{X}$ is called a standard Gale diagram. One way of obtaining standard Gale diagrams obviously consists in replacing each non-zero member $\bar{V}$ of $\bar{v}$ by $\bar{v} /\|\bar{v}\|$.

As an application we may consider the case of a $d$-polytope with $d+2$ vertices. Then its Gale transform will be in $E^{1}$ and its standard Gale diagram will be supported by the set $\{-1,0,1\}$ (compare Figure 6, where the integers $m_{i}$ indicate multiplicities, and the restrictions result from Theorem 3.2). Using standard Gale diagrams the determination of $c(d+2, d)$ and $c_{s}(d+2, d)$ becomes quite trivial.

In the most rewarding application of Gale diagrams we consider $d$-polytopes with $d+3$ vertices. Then the standard Gale diagram consists of $d+3$ points distributed on the unit circle and its center in such a manner that each open half-circle contains at least 2 points (counted with proper multiplicities). The special feature of this case is the possibility of replacing each diagram by one in which all the non-zero points are at ends of equally-spaced diameters of the circle. Moreover, one may insist to use either the least, or else the largest, possible number of those diameters, thus obtaining the "contracted standard Gale diagrams" or the "distended standard Gale diagrams." Since either type of diagram may easily be independently characterized, this leads to the possibility of carrying out the enumerations mentioned above. For details the reader should consult Grünbaum [1967a, §6.3], McMullen [1968b], or McMullen-Shephard [1970b]. A straightforward application of Pólya's [1937] enumeration theorem either to the contracted, or to the distended, standard Gale diagrams of simplicial $d$-polytopes leads to a determination of $c_{s}(d+3, d)$

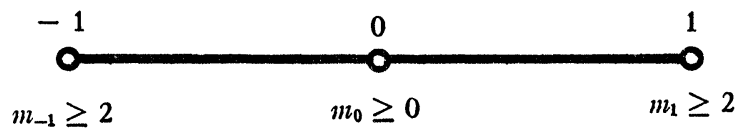

Figure 6.

(M. A. Perles, see Grünbaum [1967a, \$6.3]). A rather more involved reasoning which deals with contracted diagrams and parallels the method of the proof of Pólya's theorem, leads to an explicit formula for $c(\mathrm{~d}+3, d)$ (Lloyd [1970]). 
Similar reductions-from Gale transforms to Gale diagrams or to standard Gale diagrams-are possible mutatis mutandis for some of the variants of Gale transforms discussed at the end of $\$ 3.1$. Whenever possible, they lead to simplified enumerations-among them some rather surprising ones. The reader is urged to consult McMullenShephard [1968a], [1970a], McMullen [1968b], [1970e], and Shephard [1970a].

3.3. Projectively unique polytopes. $A d$-polytope $P$ (and its isomorphism type) are called (see Grünbaum [1967a, p. 68]) projectively unique provided every $d$-polytope $P^{\prime}$ isomorphic to $P$ is even projectively equivalent to $P$ (that is, $P^{\prime}$ is the image of $P$ under a nonsingular projective transformation).

It is not hard to prove that if a $d$-polytope is projectively unique so is each polytope dual to it, and that each $d$-polytope with at most $d+2$ vertices (or else, dually, with at most $d+2$ facets) is projectively unique. The cartesian product $T_{1} \times T_{2}$ of two simplices is projectively unique, though in general the cartesian product $P_{1} \times P_{2}$ of projectively unique polytopes $P_{i}$ is not necessarily projectively unique. For $d=2$ no polygon with 5 or more vertices (or edges) is projectively unique. The first nontrivial result in this direction concerns the case $d=3$; we have (see Grünbaum [1967a, p. 68]):

THEOREM 3.6. $A$ 3-polytope $P$ is projectively unique if and only if $P$ has at most 9 edges.

There are only 4 different types of 3 -polytopes with 9 or fewer edges (see Figure 7), and it is easy to check that they are projectively unique. It is also easy to check that

$$
\text { each of the types with } 10,11 \text {, or } 12
$$
edges is not projectively unique.

(There are 2 different types with 10 edges, 4 with 11 , and 12 with 12 edges; see, for example, Federico [1969] and the references given there. Compare Figure 7.) To complete the proof we invoke Steinitz's Theorem 1.8, and its proof as presented in Grünbaum [1967a, \$13.1]. In the terminology and notation used there, it is enough to observe that

(i) if a 3-polytope $P$ is obtained from a 3-polytope $\widetilde{P}$ by reversing one of the reductions $\omega_{i}$ or $\eta_{i}, i=0,1,2,3$, and if $\widetilde{P}$ is not projectively unique, then $P$ is not projectively unique; and

(ii) each 3-polytope $P$ with at least least 13 edges is obtainable in such a fashion from some $\widetilde{P}$ which (by induction and $\left(^{*}\right)$ ) is not projectively unique. 


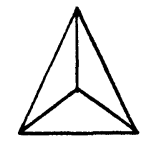

$f_{1}=6$

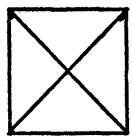

8

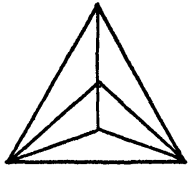

9

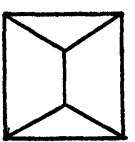

9

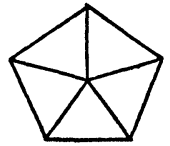

10

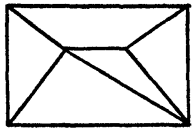

10

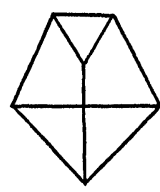

11

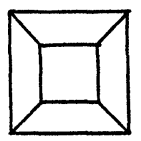

12

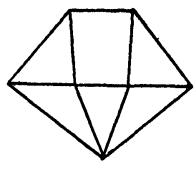

12

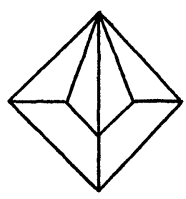

12

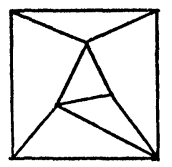

12

Figure 7.

Another result on projectively unique $d$-polytopes characterizes those which have $d+3$ vertices; it is due to M. A. Perles (see Grünbaum [1967a, p. 120], McMullen [1968a], [1968b]) and is a simple consequence of general results on Gale transforms:

Theorem 3.7. $A$ d-polytope with $d+3$ vertices is projectively unique 
if and only if its contracted standard Gale diagram has one of the forms indicated in Figure 8, where the non-negative multiplicities $m_{i}$ are integers satisfying the indicated conditions and $\sum_{i \geq 0} m_{i}=d+3$.

Theorem 3.7 and an inspection of the list of 4-polytopes with 7 vertices (see Figure 5) show that there is no analogue of Theorem 3.6 for 4 -polytopes. Indeed, $(7,17,18,8)$ is the $f$-vector of 4 different isomorphism types of 4-polytopes, of which one is projectively unique while the other three are not.
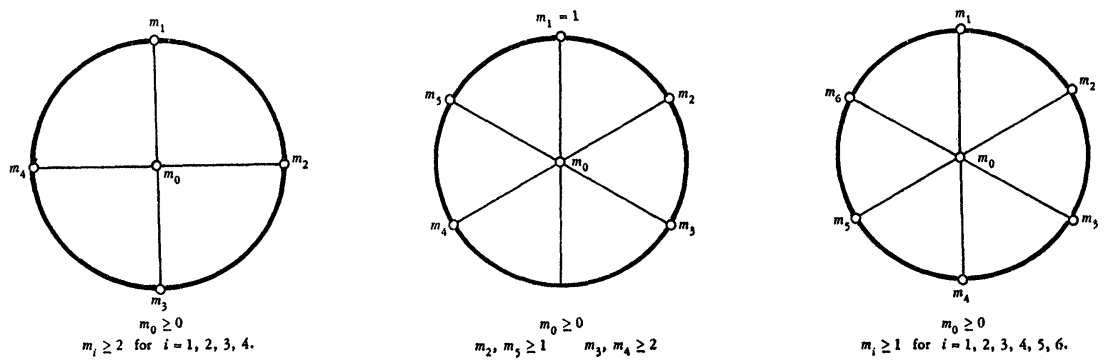

Figure 8.

In the case of 4-polytopes, the results mentioned above lead to 11 different projectively unique types. Besides the 4 -simplex and the 4 types of 4-polytopes with 6 vertices and their duals (the 2-fold 4pyramid over a quadrangle being self-dual) they are the two polytopes with 7 vertices having the Gale diagrams shown in Figure 9, and the dual of the second (the first being self-dual).

It may be conjectured that there are no other types of projectively unique 4 -polytopes. However, for each $d \geqq 4$ we are unable to settle even the

CONJECTURE 3.1. For each dimension $d$ there are only finitely many different types of projectively unique $d$-polytopes.

One of the reasons for the apparent difficulty of this conjecture seems to be related to the fact (Grünbaum [1967a, pp. 96, 208]) that a projectively unique polytope may have facets which are not projectively unique. This fact also implies that Theorem 1.11 does not generalize to higher dimensions. ${ }^{19}$

McMullen [1968a], [1968b] studied in some detail constructions which yield projectively unique polytopes from given polytopes with that property. Among results he obtained we mention here only the fact that if the projectively unique polytopes $P_{1}$ and $P_{2}$ are contained

${ }^{19}$ See Note 15 on pages $1184-1185$. 
in skew flats then their join (that is, $\operatorname{conv}\left(P_{1} \cup P_{2}\right)$ ) is also projectively unique.
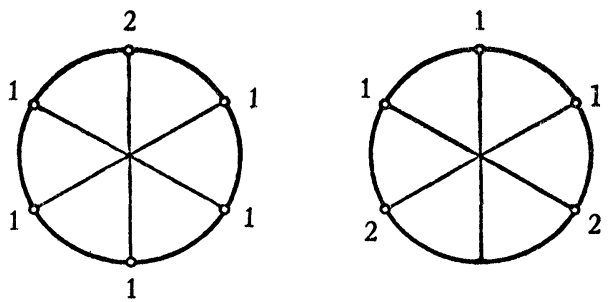

FIGURE 9.

For centrally symmetric $d$-polytopes $P$ it is rather natural to consider a notion related to projective uniqueness by requiring, in the definition of projectively unique polytopes, the polytopes $P^{\prime}$ of the definition to be centrally symmetric. (Then the projective equivalence of $P$ and $P^{\prime}$ is already a linear equivalence.) Polytopes having this property were investigated by McMullen [1969] who called them "linearly stable" and characterized them as convex hulls of centrally symmetric subsets of the set of vertices of the regular $d$-cube.

Analogous classes of polytopes may be defined with respect to any group of symmetries of $d$-polytopes; however, no other results seem to be known.

3.4. Irrational polytopes. One of the greatest triumphs of the technique of Gale diagrams is the negative solution by M. A. Perles to the following problem of Victor Klee (see Grünbaum [1967a, §5.5]):

Does every isomorphism type of $d$-polytopes have representatives all vertices of which are at points of $E^{d}$ having only rational coordinates?

As has been mentioned above (Theorem 1.9) the solution of Klee's problem is affirmative if $d=3$ (it is obviously so for $d=2$ ). Using Gale diagram techniques M. A. Perles was able to establish the following two results which complement each other in a certain sense.

TheOREM 3.8. Each $d$-polytope $P$ with at most $d+3$ vertices may be arbitrarily closely approximated by polytopes of the same isomorphism type as $P$ and having vertices at rational points of $E^{d}$.

THEOREM 3.9. There exists an 8-polytope $P$ with 12 vertices which is essentially irrational; that is, each polytope $P^{\prime}$ isomorphic to $P$ has some vertex at a point not all coordinates of which are rational.

The proof of Theorem 3.8 is very easy using general properties of 
the Gale diagrams. The proof of Theorem 3.9 uses Gale diagrams together with the fact that there exists a 2-arrangement of 9 lines and 9 points in the real (projective) plane which is not realizable using only points with rational coordinates. The details may be found in Grünbaum [1967a, §5.5]; the same method yields the analogous result in all dimensions $\geqq 8$. In dimensions 4 to 7 the question of existence of essentially irrational polytopes is still open.

Another unsolved problem that should be mentioned in this context is:

Conjecture 3.2. For each $d$ there exists a finite extension field $Q_{d}=Q\left(\alpha_{1}, \cdots, \alpha_{r_{d}}\right)$ of the field $Q$ of rationals such that every isomorphism type of $d$-polytopes is realizable by a $d$-polytope in the $d$ dimensional linear space $Q_{d}^{d}$ over the field $Q_{d}$.

V. Klee (private communication) raised also the question whether every isomorphism type of polytopes in $d$-dimensional linear spaces over a (not necessarily archimedean) ordered field is realizable in the real $d$-space. At least for $d=3$ an affirmative answer may be conjectured. ${ }^{20}$

A related open question concerns the effective enumerability of rational $d$-polytopes with $v$ vertices. No algorithm is known for deciding whether a given (real) polytope is isomorphic to a rational one.

Rationality questions are of interest in connection with other results on polytopes as well. As an example we may mention the following result of M. A. Perles (see Grünbaum [1967a, §5.1]): For each $d$-polytopes $P \subset E^{d}$ with $f$ facets, for each $(f-1)$-simplex $T \subset E^{f-1}$, and for every point $p \in$ int $T$, there exists a $d$-flat $L$ such that $p \in L$ and $L \cap T$ is affinely equivalent to $P$. This result fails to hold already for $d=2$ and $f=4$ if we deal with rational spaces instead of reals ones.

A problem similar to Klee's was posed some years ago by I. J. Schoenberg in private communications. Schoenberg asked whether each polygon may be approximated arbitrarily closely by totally rational polygons having the same number of sides. Here a polygon is called totally rational provided its edges and diagonals all have rational lengths. The answer is known (Besicovitch [1959], Mordell [1960], Daykin [1963]) to be affirmative for triangles and for quadrilaterals, even if the approximating polygons are required to have rational area as well. For some related results, and for additional

${ }^{20}$ See Note 16 on page 1185. 
reference to the quite voluminous literature, see Sheng [1966], Hadwiger-Debrunner [1960], Hadwiger-Debrunner-Klee [1964], Ang-Daykin-Sheng [1969].

It seems that it is an open problem whether each convex $n$-gon $(n \geqq 4)$ may be approximated by totally rational $n$-gons having vertices at rational points. For $n=3$ an affirmative solution may easily be obtained from the example of Müller [1953] of a dense set of points on the unit circle, all having rational coordinates and rational mutual distances.

An affirmative solution to both Schoenberg's problem and the present one would obviously follow from a positive answer to the following question of P. Erdös (see Hadwiger [1958], Ulam [1960]): Does there exist a dense subset of the plane such that all the distances between its points are rational?

Another open problem is: Can every $d$-simplex be approximated by $d$-simplices having rational contents of all faces of dimensions $1,2, \cdots, d-1, d$ ? The answer is easily seen to be affirmative if only the edge-lengths are required to be rational.

It is obvious that all the above problems have analogues for $d$ arrangements of hyperplanes (in projective $d$-space). However, no results seem to be known beyond the fact (Grünbaum [1967a, §5.5]) that already for $d=2$ there exist essentially irrational $d$-arrangements.

A different rationality problem concerning certain 3-polytopes was considered by Sansone [1928].

The results and problems discussed so far and dealing with rational polytopes are part of the more general question concerning the relationship of similarly defined objects in different settings. We would like to mention a few more instances of such parallel theories since we feel that the understanding and clarification of the differences are very important.

In Chapter 2 we have already mentioned the fact that there exist triangulations of the 3-sphere which are not isomorphic to the boundary complex of any 4-polytope. More generally and more precisely, let $\mathfrak{C}$ be a simplicial complex which is realizable by a complex the underlying set of which is a $d$-sphere. Then there exist a number of a priori possible ways of realizing $\mathfrak{C}$; in particular, $\mathfrak{C}$ may be isomorphic to

(a) the boundary complex of a simplicial $(d+1)$-polytope.

(b) a spherical-geometric complex. (A spherical-geometric complex is a triangulation of the sphere by spherically convex simplices.) 
(c) a geometric complex in $E^{d+1}$, the underlying set of which is star-shaped (that is, visible from a suitable point 0 ).

(d) a geometric complex in $E^{d+1}$.

(e) no geometric complex in $E^{d+1}$.

It is easily seen that the generality increases as we pass from (a) to (b) to (c) to (d); but the inclusion is not strict since it may be shown that the class (b) of complexes coincides with class (c). Also, it follows from the results discussed in $\$ 1.2$ that for $d=2$ the classes (a), (b), (c), and (d) coincide, while (e) is empty. For $d \geqq 3$ it is known that there exist complexes $\mathcal{C}$ in one of the classes (d) and (e) which are not in class (a). (For this result and related-material see Cairns [1940], van Kampen [1941], Grünbaum [1967a, Chapter 11], [1970b], [1970c], Grünbaum-Sreedharan [1967], Barnette [1970b], [1970e] Barnette-Wegner [1970].) ${ }^{21}$ While any additional information on the relations between the various classes would be of interest, probably the most tantalizing is the following special case of Conjecture 1.2: The class (e) is empty for each $d$.

Completely analogous problems arise if one considers centrally symmetric triangulations of the $d$-sphere, and restricts the classes (a) to (e) by requiring that all the objects considered be centrally symmetric. The results known in this case parallel those mentioned above (see Grünbaum [1970b], [1970c], [1970d], [1970e]).

In some cases it is possible to use Gale diagram methods to show that a certain complex does not belong to the class (a). For example, results of McMullen-Shephard [1968a] on the possible degree of neighborliness of centrally symmetric polytopes (obtained by the use of centrally-symmetric diagrams) show that certain triangulations of the 3-sphere (Grünbaum [1970b], [1970c], [1970d]) are not isomorphic to the boundary complex of any centrally symmetric polytope.

Another pair of parallel theories concerns $d$-arrangements. For simplicity, and also because of scarcity of results in higher dimensions, we shall discuss only the case $d=2$. Then, besides the 2 -arrangements of lines as defined in $\$ 3.1$, following Levi [1926] one may consider arrangements of pseudolines. An arrangement of pseudolines is a finite family of simple closed curves in the real projective plane, such that any two curves have one point in common at which they cross each other. While arrangements of pseudolines share many properties with arrangements of lines, not every one is "stretchable" -that is, isomorphic to an arrangement of lines. Explicit examples

\footnotetext{
${ }^{21}$ See Note 14 on page 1184.
} 
for this observation of Levi [1926] were given by Ringel [1956] (see also Grünbaum [1970d], where a more detailed discussion of the topic is given). However, despite some recent work by Kelly-Rottenberg [1970] and earlier results of Ringel [1957], the precise delineation of the properties distinguishing arrangements of pseudolines from those of lines still eludes us.

Notes Added in Proof (September 15, 1970). During the few months since the manuscript of the present paper was completed, quite a few rather remarkable achievements were published, or brought to my attention. With the aim of making this survey as upto-date as possible, the principal new results are briefly mentioned in the following notes.

1. Steinitz's theorem is also the core of the characterization of lattices isomorphic to lattices of faces of 3-polytopes, given by Altshuler [1970].

2. For a related result see Reidemeister-Horneffer [1968].

3. In a far-reaching generalization of these results Mani [1970e] has proved that every 3-polytopal graph $G$ may be realized by the 1-skeleton of a suitable 3-polytope $P$ such that each automorphism of $G$ is induced by an automorphism of $P$.

4. Analogues of Eberhard's theorem for polytopes with a center (or line, or plane) of symmetry have been established by Zaks [1970b]. The same paper provides also negative solutions to some problems of Motzkin [1967c].

5. For results analogous to Eberhard's theorem but dealing with maps on the torus see Zaks [1970c] and Barnette-Jucovič-Trenkler [1970].

6. According to R. A. Duke (private communication) the function $c(n)$ exists for each positive integer $n$ and has values satisfying

$$
\begin{aligned}
4 & \leqq c(1) \\
{\left[\frac{1}{2}(5+\sqrt{ }(16 n+1))\right] } & \leqq c(n) \leqq[4+\sqrt{ }(6 n+3)] \text { for } n>1 .
\end{aligned}
$$

7. The early proofs of Euler's theorem assumed tacitly the shellability of the boundary complex of each polytope. A surprisingly simple proof of the shellability of those complexes was recently found by Bruggesser-Mani [1970] (thus vindicating those early approaches to Euler's theorem); their method of shelling was found very useful also in McMullen's [1970g] proof of the upper-bound conjecture (see Note 8 below).

8. In July 1970 a complete proof of the upper-bound conjecture for convex polytopes was obtained by P. McMullen [1970g]; the surprisingly simple proof combines the shelling method of Bruggesser-Mani [1970], and the use of the numbers $g_{i}$ rather than work- 
ing directly with the $f_{j}^{\prime}$ 's. It is very tempting to speculate about the validity of the upper-bound conjecture, or of the $g$-conjecture discussed below, for triangulated spheres.

9. For some related investigations see Derry [1968].

10. In another surprising development, D. Barnette (private communication) proved in July 1970 the lower-bound conjecture for the number of facets $\left(\varphi_{d-1}^{*}(v, d)=(d-1) v-(d+1)(d-2)\right)$ for all $v>d \geqq 1$.

11. The numbers $g_{i}$ seem to be destined to play an important role in future developments of the combinatorial theory of polytopes, although an intuitive explanation for their relevance is still lacking. P. McMullen (private communication) has involved them in the following, rather intriguing $g$-conjecture.

For positive integers $a, k$, and $j$ let

$$
a=\left(\begin{array}{c}
a_{k} \\
k
\end{array}\right)+\left(\begin{array}{c}
a_{k-1} \\
k-1
\end{array}\right)+\cdots+\left(\begin{array}{c}
a_{i} \\
i
\end{array}\right),
$$

where $a_{k}>a_{k-1}>\cdots>a_{i+1}>a_{i} \geqq i \geqq 1$ (compare Theorem 2.7), and let

$$
a^{\langle j ; k\rangle}=\left(\begin{array}{c}
a_{k}+j-k \\
j
\end{array}\right)+\left(\begin{array}{c}
a_{k-1}+j-k \\
j-1
\end{array}\right)+\cdots+\left(\begin{array}{c}
a_{i}+j-k \\
i+j-k
\end{array}\right) .
$$

Then, in extending the "generalized lower-bound conjecture for simplicial d-polytopes" McMullen conjectured that the $f$-vectors of simplicial d-polytopes are precisely those which satisfy the inequalities

$$
0 \leqq g_{j} \leqq g_{k}^{\langle i+1 ; k+1\rangle}
$$

for all $0 \leqq k \leqq j \leqq\left[\frac{1}{2} d\right]-1$.

The inequalities in case $k=0$ of this conjecture are true, since they are just the upper-bound conjecture proved by McMullen [1970g]. McMullen has also proved the conjecture for $d \leqq 5$, as well as for $f_{0} \leqq d+2$, and for $f_{0}=d+3$ if $d \leqq 8$.

12. The analogue for cubical complexes of the Kruskal-Katona theorem was recently established in full generality by Lindström [1970b].

13. Bounds for angle-sums of different representatives of the same isomorphism type of polytopes were given by Barnette [1970f].

14. In an additional application of Gale diagram techniques Shephard [1970b] found necessary and sufficient conditions for a spherical-geometric complex on the $(d-1)$-sphere $S^{d-1}$ to be the radial projection of the boundary complex of a $d$-polytope. This extends and explains the result of Supnick [1951], who found a solution 
of the problem for $S^{2}$.

15. A construction of M. A. Perles (private communication) yields projectively unique $d$-polytopes such that the numbers of both vertices and facets are of the order of $c \cdot 3^{d / 6}$ for some constant $c$ near to 1 .

16. Lindström [1970a] gave an affirmative answer to Klee's question by proving that every polytope in a linear space over a (not necessarily archimedean) ordered field is isomorphic to a polytope in a real Euclidean space $E^{d}$.

\section{REFERENCES ${ }^{22}$}

G. L. Alexanderson and J. E. Wetsel, 1970. Dissection of a tetrahedron. [p. 1171]

C. B. Allendoerfer and A. Weil,

1943. The Gauss-Bonnet theorem for Riemannian polyhedra, Trans. Amer. Math.

A. Altshuler, Soc. 53 (1943), 101-129. MR 4, 169. [p. 1162]

1969. Polyhedral realizations in $R^{3}$ of triangulations of the torus and 2-manifolds in convex 4-polytopes, Ph.D. Thesis, Hebrew University, Jerusalem, 1969. (Hebrew) [p. 1138]

1970. Lattice characterization of convex 3-polytopes and of polygonizations of 2manifolds, Israel J. Math. 8 (1970), 57-64. [p. 1183]

D. D. Ang, D. E. Daykin and T. K. Sheng,

1969. On Schoenberg's rational polygon problem, J. Austral. Math. Soc. 9 (1969), 337-344. MR 39\#6816. [p. 1181]

Anonymous,

1964. Programma van jaarlijkse Prijsvragen, Nieuw Arch. Wisk. (3) 12 (1964), 60-65. [p. 1142]

M. L. Balinski,

1961. On the graph structure of convex polyhedra in n-space, Pacific J. Math. 11 (1961), 431-434. MR 23 \#A4059. [p. 1134]

T. Banchoff,

1967. Critical points and curvature for embedded polyhedra, J. Differential Geometry 1 (1967), 245-256. MR 37 \#921. [p. 1159, p. 1162]

R. Bantegnie,

1965. Espaces de formes affines, C. R. Acad. Sci. Paris 261 (1965), 2554-2556. MR 32 \#4677. [p. 1163]

D. W. Barnette,

1966. Trees in polyhedral graphs, Canad. J. Math. 18 (1966), 731-736. MR 33 \#3951. [p. 1147]

1967. A necessary condition for d-polyhedrality, Pacific J. Math. 23 (1967), 435440. MR 36 \#1353. [p. 1135]

1969a. On p-vectors of 3-polytopes, J. Combinatorial Theory 7 (1969), 99-103. MR 39 \#6165. [p. 1141]

1969b. A simple 4-dimensional nonfacet, Israel J. Math. 7 (1969), 16-20. MR 39 \#4745. [p. 1146, p. 1611]

${ }^{22}$ Numbers in square brackets at the end of each reference denote the pages on which that reference is quoted. 
1970a. A completely unambiguous 5-polyhedral graph, J. Combinatorial Theory 9 (1970), 44-53. [p. 1136]

1970b. Diagrams and Schlegel diagrams, Combinatorial Structures and their Applications, Gordon and Breach, New York, pp. 1-4. [p. 1137, p. 1156, p. 1182]

1970c. The graphs of polytopes with involutory automorphisms, Israel J. Math. (to appear). [p. 1139]

1970d. Projections of 3-polytopes, Israel J. Math. 8 (1970), 304-308. [p. 1139]

1970e. On the enumeration of combinatorial spheres and triangulations of the 3-sphere (to appear). [p. 1137, p. 1182]

1970f. The sum of the solid angles of a d-polytope. (to appear) [p. 1184]

D. W. Barnette and B. Grünbaum,

1969. On Steinitz's theorem concerning convex 3-polytopes and on some properties of 3-connected graphs, Lecture Notes in Math., vol. 110, Springer-Verlag, Berlin, 1969, pp. 27-40. [p. 1137]

1970. Preassigning the shape of a face, Pacific J. Math. 32 (1970), 299-306. [p. 1139]

D. W. Barnette and E. Jucovic,

1970. Hamiltonian circuits on 3-polytopes, J. Combinatorial Theory 9 (1970), 54-59. [p. 1145]

D. Barnette, E. Jucovič and M. Trenkler,

1970. Toroidal maps with prescribed types of vertices and faces. (to appear). [p. 1183]

D. W. Barnette and G. Wegner,

1970. A 3-sphere that is not 4-polyhedral (to appear). [p. 1137, p. 1156, p. 1182]

C. Berg,

1969a. Corps convexes et potentiels sphériques, Mat.-Fys. Medd. Danske Vid. Selsk. 37 (1969), no. 6. [p. 1163]

1969b. Abstract Steiner points for convex polytopes, Copenhagen University Math. Institut, Preprint Series, no. 6, 1969. [p. 1163]

A. J. Bernstein,

1967. Maximally connected arrays on the n-cube, SIAM J. Appl. Math. 15 (1967), 1485-1489. MR 36 \#6308. [p. 1156]

A. S. Besicovitch,

1959. Rational polygons, Mathematika 6 (1959), 98. MR 22 \#1557. [p. 1180]

W. Blaschke,

1915. Einige Bemerkungen über Kurven und Flächen konstanter Breite, Ber. Verh. Sächs. Akad. Wiss. Leipzig. Math.-Nat. Kl. 67 (1915), 290-297. [p. 1165]

E. D. Bolker,

1969. A class of convex bodies, Trans. Amer. Math. Soc. 145 (1959), 323-345. [p. 1170]

1970. Centrally symmetric polytopes, Proc. Twelfth Biennial Internat. Seminar of the Canadian Math. Congress (Vancouver, B.C., 1969) Canad. Math. Soc. Montreal 1970. (to appear). [p. 1170]

B. Bollobas,

1967. Fixing systems for convex bodies, Studia Sci. Math. Hungar. 2 (1967), 351354. MR 36 \#3230. [p. 1173]

W. E. Bonnice and L. M. Kelly,

1970. On the number of ordinary planes (to appear). [p. 1171]

W. Bonnice and V. Klee,

1963. The generation of convex hulls, Math. Ann. 152 (1963), 1-29. MR 27 \#6108. [p. 1171]

W. E. Bonnice and J. R. Reay, 
1969. Relative interiors of convex hulls, Proc. Amer. Math. Soc. 20 (1969), 246-250. MR 38 \#2669. [p. 1171]

J. Bosák,

1967. Hamiltonian lines in cubic graphs, Proc. Internat. Sympos. Theory of Graphs (Rome, 1966) Gordon and Breach, New York; Dunod, Paris, 1967, pp. 35-46. MR $36 \# 5022$. [p. 1144]

R. Bott,

1952. Two new combinatorial invariants for polyhedra, Portugal. Math. 11 (1952), 35-40. MR 14, 74. [p. 1157]

G. Böttger and H. Harders,

1964. Note on a problem by S. L. Hakimi concerning planar graphs without parallel elements, J. Soc. Indust. Appl. Math. 12 (1964), 838-839. MR 30 \#2481. [p. 1142]

H. Bowen and S. Fisk,

1967. Generation of triangulations of the sphere, Math. Comp. 21 (1967), 250-252. MR 36 \#6325. [p. 1173, p. 1174]

T. A. Brown,

1960. The representation of planar graphs by convex polyhedra, Note P-2085, The Rand Corp., Santa Monica, Calif., 1960. [p. 1138]

1961. Simple paths on convex polyhedra, Pacific J. Math. 11 (1961), 1211-1214. MR 25 \#3864. [p. 1147]

W. G. Brown,

1965. Historical note on a recurrent combinatorial problem, Amer. Math. Monthly 72 (1965), 973-977. [p. 1173]

M. Brückner,

1893. Die Elemente der vierdimensionalen Geometrie mit besonderer Berücksichtigung der Polytope, Jber. Ver. Naturkunde Zwickau 1893, 61 pp. [p. 1173]

1909. Über die Ableitung der allgemeinen Polytope und die nach Isomorphismus verschiedenen Typen der allgemeinen Achtzelle (Oktatope), Verh. Konink. Akad. Wetensch. 10 (1909), no. 1, 29 pp. [p. 1137, p. 1173]

H. Bruggesser and P. Mani,

1970. Shellable decompositions of cells and spheres. (to appear). [p. 1183]

S. S. Cairns,

1940. Triangulated manifolds which are not Brouwer manifolds, Proc. Nat. Acad. Sci. U.S.A. 26 (1940), 359-361. MR 1, 317. [p. 1182]

R. J. Canham,

1969. A theorem on arrangements of lines in the plane, Israel J. Math. 7 (1969), 393-397. [p. 1171]

C. Carathéodory,

1907. Ueber den Variabilitätsbereich der Koeffizienten von Potenzreihen, die gegebene Werte nicht annehmen, Math. Ann. 64 (1907), 95-115. [p. 1150]

L. Carlitz,

1961. The sum of the angles in an n-dimensional simplex, Amer. Math. Monthly 68 (1961), 901-902. MR 24 \#A477. [p. 1157]

A. Cayley,

1862. On the $\Delta$-faced polyacrons in reference to the problem of the enumeration of polyhedra, Mem. Lit. Philos. Soc. Manchester 1 (1862), 248-256; Coll. Math. Papers 5 (1892), 38-43. [p. 1135]

J. L. Chrislock,

1966. Imbedding a skeleton of a simplex in euclidean space, Amer. Math. Monthly 73 (1966), 381-382. [p. 1134] 
J. Chuard,

1932. Les réseaux cubiques et le problème des quatres couleurs, Mém. Soc. Vaudoise Sci. Nat. 4 (1932), 41-101. [p. 1143]

1966. Graphes planaires homogènes de degre 3, J. Combinatorial Theory 1 (1966), 411-436. MR 34 \#4157. [p. 1147]

V. Chvátal,

1969. Planarity of graphs with given degrees of vertices, Nieuw Arch. Wisk. 17 (1969), 47-60. [p. 1142, p. 1143]

G. F. Clements,

1970. Sets of lattice points which contain a maximal number of edges (to appear). [p. 1156]

G. F. Clements and B. Lindström,

1969. A generalization of a combinatorial theorem of Macauley, J. Combinatorial Theory 7 (1969), 230-238. MR 40 \#50. [p. 1156]

J. L. Coolidge,

1940. A history of geometrical methods, Oxford Univ. Press, New York, 1940. MR 2, 113. [p. 1132]

H. S. M. Coxeter,

1962. The classification of zonohedra by means of projective diagrams, J. Math. Pures Appl. (9) 41 (1962), 137-156; reprinted in Twelve geometric essays, Southern Illinois Univ. Press, Carbondale, Ill., 1968, pp. 54-74. MR 25 \#4417. [p. 1171]

D. W. Crowe,

1969. Nearly regular polyhedra with two exceptional faces, Lecture Notes in Math. vol. 110, Springer-Verlag, Berlin, 1969, pp. 63-76. [p. 1143]

D. W. Crowe and J. Molnár,

1969. On polyhedra with specified types of face, Math. Gaz. 53 (1969), 45-50. [p. 1143]

N. C. Dalkey,

1967. Parity patterns on even triangulated polygons, J. Combinatorial Theory 2 (1967), 100-102. MR 34 \#4159. [p. 1142]

L. Danzer, B. Grünbaum and V. Klee,

1963. Helly's theorem and its relatives, Proc. Sympos. Pure Math., vol. 7, Amer. Math. Soc., Providence, R. I., 1963, pp. 101-180. MR 28 \#524. [p. 1171]

C. Davis,

1954. Theory of positive linear dependence, Amer. J. Math. 76 (1954), 733-746. MR 16, 211. [p. 1171]

D. E. Daykin,

1963. Rational polygons, Mathematika 10 (1963), 125-131. MR 30 \#63. [p. 1180]

H. Debrunner,

1955. Zu einem massgeometrischen Satz uber Körper konstanter Breite, Math. Nachr. 13 (1955), 165-167. MR 17, 294. [p. 1165]

D. Derry,

1968. Polygons of order $n$ in $L_{n}$ with $n+2$ vertices, Math. Scand. 23 (1968), 73-95.

A. Dinghas, [p. 1184]

1940. Verallgemeinerung eines Blaschkeschen Satzes über konvexe Körper konstanter R. A. Duke, Breite, Rev. Math. Union Interbalkan. 3 (1940), 17-20. MR 2, 261. [p. 1165]

1970. Geometric embedding of complexes, Amer. Math. Monthly 77 (1970), 597603. [p. 1138] 
V. Eberhard,

1891. Zur Morphologie der Polyeder, Teubner, Leipzig, 1891. [p. 1138; p. 1140]

H. G. Eggleston, B. Grünbaum and V. Klee,

1964. Some semicontinuity theorems for convex polytopes and cell-complexes, Comment. Math. Helv. 39 (1964), 165-188. MR $30 \# 5217$. [p. 1152]

G. Ewald,

1965. Von Klassen konvexer Körper erzeugte Hilberträume, Math. Ann. 162 (1965/ 66), 140-146. MR 32 \#8255. [p. 1163]

G. Ewald and G. C. Shephard,

1966. Normed vector spaces consisting of classes of convex sets, Math. Z. 91 (1966), 1-19. MR 32 \#4597. [p. 1163]

L. Euler,

1752a. Elementa doctrinae solidorum, Comment. Acad. Sci. Imp. Petrop. 4 (1752/ 53), 109-140. [p. 1173]

1752b. Demonstratio nonullarum insignium proprietatum, quibus solida hedris planis inclusa sunt praedita, Comment. Acad. Sci. Imp. Petrop. 4 (1752/53), 140160. [p. 1173]

I. Fáry,

1948. On straight line representation of planar graphs, Acta Univ. Szeged. Sect. Sci. Math. 11 (1948), 229-233. MR 10, 136. [p. 1138]

1949. Sur la courbure totale d'une courbe gauche faisant un noeud, Bull. Soc. Math. France 77 (1949), 128-138. MR 11, 393. [p. 1159]

1960. Translation invariant, additive functionals related to mixed volumes, ONR Technical Report, Berkeley, 1960. [p. 1166]

1961. Functionals related to mixed volumes, Illinois J. Math. 5 (1961), 425-430. MR 24 \#A811. [p. 1166]

P. J. Federico,

1969. Enumeration of polyhedra: The number of 9-hedra, J. Combinatorial Theory 7 (1969), 155-161. MR 39 \#446. [p. 1174, p. 1176]

L. Fejes Tóth,

1962. On primitive polyhedra, Acta Math. Acad. Sci. Hungar. 13 (1962), 379-382. MR 26 \#2942. [p. 1171]

W. J. Firey and B. Grünbaum,

1964. Addition and decomposition of convex polytopes, Israel J. Math. 2 (1964), 91-100. MR 30 \#5218. [p. 1163, p. 1171]

H. Flanders,

1966. The Steiner point of a closed hypersurface, Mathematika 13 (1966), 181-188. MR 34 \#6695. [pp. 1162, 1163]

A. Flores,

1932. Über die Existenz $n$-dimensionaler Komplexe, die nicht in den $R_{2 n}$ topologisch einbettbar sind, Ergebnisse Math. Kolloq. 5 (1932), 17-24. [p. 1135]

1933. Über $n$-dimensionale Komplexe, die im $R_{2 n+1}$ absolut selbstverschlungen sind, Ergebnisse Math. Kolloq. 6 (1933), 4-6. [p. 1135]

D. Gale,

1963. Neighborly and cyclic polytopes, Proc. Sympos. Pure Math., vol. 7, Amer. Math. Soc., Providence, R. I., 1963, pp. 225-232. MR 27 \#2915. [p. 1150]

T. Gallai,

1970. Signierte Zellenzerlegungen, Acta Math. Acad. Sci. Hungar. (to appear). [p. 1142]

D. W. Grace,

1965. Computer search for non-isomorphic convex polyhedra, Report CS 15, Computer Science Dept., Stanford University, Stanford, Calif., 1965. [p. 1173] 
E. Ja. Grinberg,

1968. Plane homogeneous graphs of degree three without Hamiltonian circuits, Latvian Math. Yearbook, 4 Izdat. "Zinatne", Riga, 1968, pp. 51-58. (Russian) MR 39 \#96. [p. 1144]

H. Grötzsch,

1956. Zur Theorie der Dreikantnetze auf der Kugel und der einfach Diskreten Gebilde. I. Elementare kombinatorische Eigenschaften gewisser punktierten Kugel, Wiss. Z. Martin-Luther-Univ. Halle-Wittenberg. Math.-Nat. Reihe 5 (1956), 839-844. MR 22 \#2987a. [p. 1143]

1962. Zur Theorie der diskreten Gebilde. 15. Mitteilung: Zusatzbemerkungen, Wiss. Z. Martin-Luther-Univ. Halle-Wittenberg. Math.-Nat. Reihe 11 (1962), 733-736. MR 29 \#1637. [p. 1143]

B. Grünbaum,

1963a. Unambiguous polyhedral graphs, Israel J. Math. 1 (1963), 235-238. MR 32 \#2972. [p. 1136]

1963b. Measures of symmetry for convex sets, Proc. Sympos. Pure Math., vol. 7, Amer. Math. Soc., Providence, R. I., 1963, pp. 233-270. MR 27 \#6187. [p. 1163]

1964. Fixing systems and inner illumination, Acta Math. Acad. Sci. Hungar. 15 (1964), 161-163. MR 28 \#3367. [p. 1173]

1965. On the facial structure of convex polytopes, Bull. Amer. Math. Soc. 71 (1965), 559-560. MR 32 \#448. [p. 1135]

1967a. Convex polytopes, Interscience, New York, 1967. MR 37 \#2085.

1967b. The number of faces of convex polytopes, Proc. Colloq. Convexity (Copenhagen, 1965) K $\phi$ benhavns Univ. Mat. Inst., Copenhagen, 1967. [p. 1131 p. 1149]

1968a. Some analogues of Eberhard's theorem on convex polytopes, Israel J. Math. 6 (1968), 398-411. MR 39 \#6168. [p. 1140, p. 1141]

1968b. Grassmann angles of convex polytopes, Acta Math. 121 (1968), 293-302. MR 38 \#6455. [p. 1159, p. 1160, p. 1161]

1969a. Planar maps with prescribed types of vertices and faces, Mathematika 16 (1969), 28-36. MR 39 \#6768. [p. 1141]

1969b. Graphs, complexes, and polytopes, Recent Progress in Combinatorics, Academic Press, New York, 1969, pp. 85-90. [p. 1138]

1969c. Some results on the upper-bound conjecture for convex polytopes, SIAM J. Appl. Math. 17 (1969), 1142-1149. [p. 1151, p. 1152]

1969d. Imbeddings of simplicial complexes, Comment. Math. Helv. 44 (1969), 502513. [p. 1135, p. 1138]

1969e. Nerves of simplicial complexes, Aequationes Math. (to appear). [p. 1157]

1970a. Some combinatorial problems, Combinatorial Structures and their Applications, Gordon and Breach, New York, 1970, pp. 500-501. [p. 1135, 1139]

1970b. On the enumeration of convex polytopes and combinatorial spheres (to appear). [p. 1137, p. 1156, p. 1182]

1970c. On combinatorial spheres, Combinatorial Structures and their Applications, Gordon and Breach, New York, 1970, pp. 119-122. [p. 1137, p. 1152, p. 1156, p. 1182]

1970d. The importance of being straight, Proc. Twelfth Biennial Internat. Seminar of the Canadian Math. Congress (Vancouver B. C., 1969) Canad. Math. Soc., Montreal, 1970. [p. 1137, p. 1156, p. 1171, p. 1182]

1970e. Arrangements, spreads, and topological planes. (to appear). [p. 1171, p. 1182]

1970f. Valence sequence and related topics. [p. 1142] 
1970g. Higher-dimensional analogues of the four-color problem and some inequalities for simplicial complexes, J. Combinatorial Theory 8 (1970), 147-153. [p. 1143, p. 1155]

B. Grünbaum and T. S. Motzkin

1962. Longest simple paths in polyhedral graphs, J. London Math. Soc. 37 (1962), 152-160. MR 25 \#2598. [p. 1146]

1963a. On polyhedral graphs, Proc. Sympos. Pure Math., vol. 7, Amer. Math. Soc., Providence, R. I., 1963, pp. 285-290. MR 27 \#2976. [p. 1135, p. 1136]

1963b. The number of hexagons and the simplicity of geodesics on certain polyhedra, Canad, J. Math. 15 (1963), 744-751. MR 27 \#4133. [p. 1141]

B. Grünbaum and G. C. Shephard,

1969. Convex polytopes, Bull. London Math. Soc. 1 (1969), 257-300. [p. 1131, p. 1139, p. 1149, p. 1168]

B. Grünbaum and V. P. Sreedharan,

1967. An enumeration of simplicial 4-polytopes with 8 vertices, J. Combinatorial Theory 2 (1967), 437-465. MR 35 \#6025; MR 35 \#6025. [p. 1135, p. 1137, p. 1156, p. 1173 , p. 1174, p. 1182$]$

R. K. Guy,

1967. Dissecting a polygon into triangles, Research Paper, no. 9, Dept. of Mathematics, University of Calgary, Calgary, Alberta, 1967. [p. 1173]

H. Hadwiger,

1957. Vorlesungen über Inhalt. Oberfä̈che und Isoperimetrie, Springer-Verlag, Berlin, 1957. MR 21 \#1561. [p. 1166]

1958. Ungelöste Probleme, No. 24, Elem. Math. 13 (1958), 85. [p. 1181]

1968. Eine Schnittrekursion für die Eulersche Charakteristik euklidischer Polyeder mit Anwendungen innerhalb der kombinatorischen Geometrie, Elem. Math. 23 (1968), 121-132. MR 38 \#5112. [p. 1149]

1969a. Zur axiomatischen Charakterisierung des Steinerpunktes konvexer Körper, Israel J. Math. 7 (1969), 168-176. [p. 1166]

1969b. Eckenkrümmung beliebiger kompakter euklidischer Polyeder und Charakteristik von Euler-Poincarê, Enseignment Math. 15 (1969), 147-151. M R 40 \#834. [p. 1162]

1970. Zur axiomatischen Charakterisierung des Steinerpunktes konvexer Körper, Israel J. Math. (to appear). [p. 1166]

H. Hadwiger and H. Debrunner,

1960. Kombinatorische Geometrie in der Ebene, Monographies de L'Enseignement Mathématiques, no. 2, Inst. Math. Univ. Genève, 1960. MR 22 \#11310. [p. 1181]

H. Hadwiger, H. Debrunner and V. Klee,

1964. Combinatorial geometry in the plane, Holt, Rinehart and Winston, New York, 1964. MR 29 \#1577. [p. 1181]

S. L. Hakimi,

1962. On the realizability of a set of integers as degrees of the vertices of a linear graph. I, II, J. Soc. Indust. Appl. Math. 10 (1962), 496-506; ibid. 11 (1963), 135147. MR $26 \# 5558$; MR 27 \#2972. [p. 1142]

R. Halin,

1966. Zu einem Problem von B. Grünbaum, Arch. Math. (Basel) 17 (1966), 566568. MR 35 \#5355. [p. 1135]

G. Hansel,

1967. Problemes de denombrement et d'evaluation de bornes concernant les elements $d u$ 
treillis distributif libre, Publ. Inst. Stat. Univ. Paris 16 (1967), 159-300. [p. 1152]

W. Hansen and V. Klee,

1969. Intersection theorems for positive sets, Proc. Amer. Math. Soc. 22. (1969), 450-457. [p. 1171]

L. H. Harper,

1964. Optimal assignments of numbers to vertices, J. Soc. Indust. Appl. Math. 12 (1964), 131-135. MR 29 \#41. [p. 1156]

K. Hauschild,

1967. Über Färbungen von 4-regulären Landkarten, Wiss. Z. Techn. Hochsch. Ilmenau 13 (1967), 399-402. MR 39 \#4048. [p. 1143]

1968. Über ein Farbungsproblem auf der Kugel, Beitrage zur Graphen theorie Internat. Kolloq. Manebach, 1967. Teubner, Leipzig, 1968, pp. 61-70. MR $40 \# 1302$. [p. 1143]

A. F. Hawkins, A. C. Hill, J. E. Reeve and J. A. Tyrrell, 1966. On certain polyhedra, Math. Gaz. 50 (1966), 140-144. [p. 1143]

P. J. Heawood,

1936. Failures in congruences connected with the four-colour map theorem, Proc. London Math. Soc. (2) 40 (1936), 189-202. [p. 1142]

F. Hering,

1969. Untersuchungen über die kombinatorische Struktur von Polyedern, Ph.D. Thesis, Bonn, 1969. [p. 1134]

O. Hermes,

1899. Die Formen der Vielfläche, J. Reine Angew. Math. 120 (1899), 27-59, 305353; ibid. 122 (1900), 124-154; 123 (1901), 312-342. [p. 1174]

W. Höhn,

1953. Winkel und Winkelsumme im n-dimensionalen euklidischen Simplex, Ph.D. Thesis, Eidgenössische Technische Hochschule, Zürich, 1953. MR 15, 55. [p. 1158]

H. F. Hunter,

1962a. On non-Hamiltonian maps and their duals, Ph.D. Thesis, Rensselaer Poly-

E. Jucovic, tech. Inst., 1962. [p. 1143]

1962. Self-conjugate K-polyhedra, Mat.-Fys. Casopis. Sloven. Akad. Vied. 12 (1962), 1-22. (Russian) MR 28 \#2478. [p. 1173]

1968. A note on paths in quadrangular polyhedral graphs, Casopis Pěst. Mat. 93 (1968), 69-72. (Slovak) MR 39 \#2658. [p. 1147]

1969. On polyhedral realizability of certain sequences, Canad. Math. Bull. 12 (1969), 31-39. MR 39 \#5390. [p. 1141]

1970a. On the number of hexagons in a map, J. Combinatorial Theory (to appear). [p. 1141]

1970b. Characterization of the p-vector of a self-dual 3-polytope, Combinatorial Structures and their Applications, Gordon and Breach, New York, 1970, pp. 185-187. [p. 1142]

1970c. On the p-vector of a 4-valent convex and toroidal 3-polytope, (to appear). [p. 1150]

H. A. Jung,

1967. Zusammenzüge und Unterteilungen von Graphen, Math. Nachr. 35 (1967), 241-267. MR 37 \#3947. [p. 1135]

1970a. A variation of $n$-connectedness, Combinatorial Structures and their Applications, Gordon and Breach, New York, 1970, pp. 189-191. [p. 1135] 
1970b. Eine Verallgemeinerung des n-fachen Zusammenhangs fur Graphen, Math. Ann. (to appear). [p. 1135]

E. R. van Kampen,

1932. Komplexe in Euklidischen Räumen, Abh. Math. Sem. Univ. Hamburg 9 (1932), 72-78. [p. 1135]

1941. Remark on the address of S. S. Cairns, Lectures in Topology, Univ. of Michigan Press, Ann Arbor, Mich., 1941, pp. 311-313. MR 3, 135. [p. 1182]

G. Katona,

1968. A theorem on finite sets, Proc. Colloq. Theory of Graphs (Tihany, 1966) Academic Press, New York; Akad. Kiad6, Budapest, 1968, pp. 187-207. [p. 1152]

L. M. Kelly and R. R. Rottenberg,

1970. Simple points in pseudoline arrangements (to appear). [p. 1183]

T. P. Kirkman,

1854. On the representation and enumeration of polyedra, Mem. Lit. Philos. Soc. Manchester (2) 12 (1854), 47-70 [p. 1173]

1857. On autopolar polyedra, Philos. Trans. Roy. Soc. London 147 (1857), 183215. [p. 1137, p. 1138]

V. Klee,

1964a. A property of d-polyhedral graphs, J. Math. Mech. 13 (1964), 1039-1042. MR 29 \#6487. [p. 1134, p. 1136]

1964b. A combinatorial analogue of Poincarê's duality theorem, Canad. J. Math. 16 (1964), 517-531. MR 32 \#6466. [p. 1151]

1964c. On the number of vertices of a convex polytope, Canad. J. Math. 16 (1964), 701-720. MR 29 \#3955. [p. 1151]

1966. Convex polytopes and linear programming, Proc. IBM Sci. Comput. Sympos. Combinatorial Problems (Yorktown Heights, N. Y., 1964) IBM Data Process. Division, White Plains, New York, 1966, pp. 123-158. MR 36 \#786. [p. 1131, p. 1149]

V. Klee and D. Walkup,

1967. The $d$-step conjecture for polyhedra of dimension $d<6$, Acta Math. 117 (1967), 53-78. MR 34 \#6639. [p. 1147]

A. Kotzig,

1965. Coloring of trivalent polyhedra, Canad. J. Math. 17 (1965), 659-664. MR 33 \#712. [p. 1142]

1970. Regularly connected 3-valent graphs without non-trivial cuts of cardinality 3 (to appear). [p. 1138]

J. B. Kruskal,

1963. The number of simplices in a complex, Mathematical Optimization Techniques, Univ. of California Press, Berkeley, Calif., 1960, pp. 251-278. MR 27 \#4771. [p. 1152]

1969. The number of s-dimensional faces in a complex: An analogy between the simplex and the cube, J. Combinatorial Theory 6 (1969), 86-89. MR 38 \#4328. [p. 1156]

D. G. Larman,

1970. Paths on polytopes, Proc. London Math. Soc. (3) 20 (1970), 161-178. [p. 1147]

D. G. Larman and P. Mani,

1970a. On the existence of certain configurations within graphs and the 1-skeletons of polytopes, Proc. London Math. Soc. (3) 20 (1970), 144-160. [p. 1134, p. 1135]

1970b. Gleichungen und Ungleichungen für Geriuste die von konvexen Polytopen und Zellenkomplexen, Comment. Math. Helv. 45 (1970), 199-218. [p. 1162] 
J. Lederberg,

1966. Systematics of organic molecules, graph theory and Hamiltonian circuits, Instrumentation Research Laboratory Report, no. 1040, Stanford University, Stanford, Calif., 1966. [p. 1144]

1967. Hamilton circuits of convex trivalent polyhedra (up to 18 vertices), Amer. Math. Monthly 74 (1967), 522-527. MR 35 \#2770. [p. 1145]

K.-C. Lee,

1959. Kombinatorische Invarianten von endlichem Komplex, Acta Math. Sinica 8 (1958), 473-482; Sci. Sinica 8 (1959), 449-460=Chinese Math. Acta 9 (1967), 199-208. MR 21 \#3834; 3835. [p. 1157]

F. Levi,

1926. Die Teilung der projektiven Ebene durch Gerade oder Pseudogerade, Ber. Verh. Sächs. Akad. Wiss. Leipzig. Math.-Nat. K1 78 (1926), 256-267. [p. 1182, p. 1183]

N. F. Lindquist,

1968. Approximation of convex bodies by finite sums of line segments, Notices Amer. Math. Soc. 15 (1968), 138. Abstract \#653-207. [p. 1170]

J. H. Lindsey, II,

1964. Assignment of numbers to vertices, Amer. Math. Monthly 71 (1964), 508-516. MR 29 \#5751. [p. 1156]

B. Lindstrom,

1970a. On the realization of convex polytopes. Euler's formula and Mobius functions, Aequationes Math. (to appear). [p. 1185]

1970b. The optimal number of faces in cubical complexes (to appear). [p. 1184]

E. K. Lloyd,

1970. The number of $d$-polytopes with $d+3$ vertices, Mathematika (to appear). [p. 1173, p. 1174, p. 1175]

L. A. Lyusternik,

1956. Convex figures and polyhedra, GITTL, Moscow, 1956; English transl., Dover, New York, 1963; Heath, Boston, Mass., 1966. MR 19, 57; MR 28 \#4427; MR 36 \#4435. [p. 1137]

I. G. Macdonald,

1970. Polynomials associated with finite cell complexes (to appear). [p. 1149] W. Mader,

1967. Homomorphieeigenschaften und mittlere Kantendichte von Graphen, Math. Ann. 174 (1967), 265-268. MR 36 \#3668. [p. 1135]

1968. Homomorphiesätze für Graphen, Math. Ann. 178 (1968), 154-168. MR 37 \#5124. [p. 1135]

J. Malkevitch,

1969. Properties of planar graphs with uniform vertex and face structure, Ph.D. Thesis, University of Wisconsin, Madison, Wis., 1969. [p. 1141, p. 1142]

1970a. Properties of planar graphs with uniform vertex and face structure, Mem. Amer. Math. Soc. No. 99 (1970). [p. 1141, p. 1142]

1970b. A survey of 3-valent 3-polytopes with two types of faces, Combinatorial Structures and their Applications, Gordon and Breach, New York, 1970, pp. 255-256. [p. 1141]

P. Mani,

1970a. Bridges in 6-connected graphs (to appear). [p. 1135]

1970b. Spheres with ferw vertices, J. Combinatorial Theory (to appear). [p. 1156]

1970c. On angle sums and Steiner points of polyhedra, Israel J. Math. (to appear). [p. 1162, p. 1166] 
1970d. On polytopes fixed by their vertices, Acta Math. Acad. Sci. Hungar. (to appear). [p 1173]

1970e. Automorphismen von polyedrischen Graphen (to appear). [p. 1183]

R. L. McKinney,

1962. Positive basis for linear spaces, Trans. Amer. Math. Soc. 103 (1962), 131148. MR $26 \# 5392$. [p. 1171]

P. McMullen,

1968a. On the combinatorial structure of convex polytopes, $\mathrm{Ph} . \mathrm{D}$. Thesis, University of Birmingham, 1968. [p. 1168, p. 1169, p. 1177, p. 1178]

1968b. Seminar on convex polytopes, Lecture Notes, Western Washington State College, Bellingham, 1968. [p. 1149, p. 1168, p. 1170, p. 1175, p. 1176, p. 1177]

1969. Linearly stable polytopes, Canad. J. Math. 21 (1969), 1427-1431. [p. 1179]

1970a. On the upper bound conjecture for convex polytopes, J. Combinatorial Theory (to appear). [p. 1150, p. 1151]

1970b. Further results on the upper-bound conjecture for convex polytopes (to appear). [p. 1152]

1970c. A lower-bound conjecture for convex polytopes (to appear). [p. 1153]

1970d. On a problem of Klee concerning convex polytopes, Israel J. Math. 8 (1970), 1-4. [p. 1153]

1970e. On zonotopes (to appear). [p. 1170, p. 1171, p. 1176]

1970f. Polytopes with centrally symmetric faces (to appear). [p. 1170]

1970g. The maximum numbers of faces of a convex polytope, Mathematika (to appear). [p. 1183, p. 1184]

P. McMullen and G. C. Shephard,

1968a. Diagrams for centrally symmetric polytopes, Mathematika 15 (1968), 123138. MR 38 \#6456. [p. 1156, p. 1168, p. 1169, p. 1176, p. 1182]

1968b. The upper bound conjecture, Lecture Notes, University of East Anglia, Norwich, 1968. [p. 1150, p. 1151, p. 1161, p. 1168]

1970a. Polytopes with an axis of symmetry, Canad. J. Math. 22 (1970), 265-287. [p. 1150, p. 1151]

1970b. The upper bound conjecture for convex polytopes, London Math. Soc. Lecture Note Series. [p. 1168, p. 1175]

1970c. Representations and diagrams (to appear). [p. 1166]

P. McMullen and D. W. Walkup,

1970. A generalized lower-bound conjecture for simplicial polytopes (to appear).

B. L. Meek, [p. 1154]

1968. Some results on k-maps, Math. Gaz. 52 (1968), 33-42. [p. 1143]

D. M. Mesner and M. E. Watkins,

1966. Some theorems about n-vertex connected graphs, J. Math. Mech. 16 (1966), 321-326. MR 34 \#94. [p. 1134]

W. Meyer,

1969. Minkowski addition of convex sets, Ph.D. Thesis, University of Wisconsin, Madison, Wis., 1969. [p. 1163]

H. Minkowski,

1897. Allgemeine Lehrsätze über konvexe Polyeder, Nachr. Ges. Wiss. Göttingen 1897, 198-219; Cf: Ges. Abh. Vol. 2, Leipzig and Berlin, pp. 103-121, 1911; reprint, Chelsea, New York, 1967. [p. 1171]

1903. Volumen und Oberfäche, Math. Ann. 57 (1903), 447-495; Cf: Ges. Abd. 
Vol. 2, Leipzig and Berlin, 1911; pp. 230-276; reprint, Chelsea, New York, 1967. [p. 1171]

J. W. Moon and L. Moser,

1963. Simple paths on polyhedra, Pacific J. Math. 13 (1963), 629-631. MR 27 \#4225. [p. 1146, p. 1147]

L. J. Mordell,

1960. Rational quadrilaterals, J. London Math. Soc. 35 (1960), 277-282. MR 23 \#A1593. [p. 1180]

T. S. Motzkin,

1964. The evenness of the number of edges of a convex polyhedron, Proc. Nat. Acad. Sci. U.S.A. 52 (1964), 44-45. MR 30 \#3411. [p. 1142]

1967a. The edge number of regular-homomorphic 3-polyhedra, Proc. Colloq. Convexity (Copenhagen, 1965) Kфbenhavns Univ. Mat. Inst., Copenhagen, 1967, pp. 212-213. [p. 1142]

1967b. The frequencies of vertex and face valences of convex and of more general 2tesselations, Proc. Colloq. Convexity (Copenhagen, 1965) Kфbenhavns Univ. Mat. Inst., Copenhagen, 1967, pp. 214-218. [p. 1142]

1967c. Combinatorial realization of centrally symmetric convex polyhedra, J. Combinatorial Theory 3 (1967), 441. [p. 1183]

A. Müller,

1953. Auf einem Kreis liegende Punktmengen ganzzahliger Entfernungen, Elem. Math. 8 (1953), 37-38. [p. 1181]

H. R. Müller,

1967. Zur axiomatischen Bergïndung der Eikörperfunktionale, Monatsh. Math. 71 (1967), 338-343. MR 36 \#5818. [p. 1166]

O. Ore,

1967. The four-color problem, Pure and Appl. Math., vol. 27, Academic Press, New York, 1967. MR 36 \#74. [p. 1146]

M. A. Perles and G. T. Sallee,

1970. Cell complexes, valuations and the Euler relation, Canad. J. Math. 22 (1970), 235-241. [p. 1166]

M. A. Perles and G. C. Shephard,

1967a. Facets and nonfacets of convex polytopes, Acta Math. 119 (1967), 113-145. MR 36 \#7022. [p. 1160, p. 1161]

1967b. Angle sums of convex polytopes, Math. Scand. 21 (1967), 199-218. MR 39

G. Polya, \#4747. [p. 1158, p. 1159, p. 1160]

1937. Kombinatorische Anzahlbestimmungen für Gruppen, Graphen und chemische Verbindungen, Acta Math. 68 (1937), 145-254. [p. 1175]

H. Rademacher,

1965. On the number of certain types of polyhedra, Illinois J. Math. 9 (1965), 361380. MR 31 \#3927. [p. 1173]

J. R. Reay,

1965a. A new proof of the Bonnice-Klee theorem, Proc. Amer. Math. Soc. 16 (1965), 585-587. MR 31 \#5145. [p. 1171]

1965b. Generalizations of a theorem of Caratheodory, Mem. Amer. Math. Soc. No. 54 (1965). MR 32 \#6319. [p. 1171]

1966. Unique minimal representations with positive bases, Amer. Math. Monthly 73 (1966), 253-261. MR 33 \#7827. [p. 1171]

1967. Positive bases as a tool in convexity, Proc. Colloq. Convexity (Copenhagen, 
1965) Kфbenhavns Univ. Mat. Inst., Copenhagen, 1967, pp. 255-260. MR 36 \#5819. [p. 1171]

1968a. Projections of f-vectors of 4-polytopes, Notices Amer. Math. Soc. 15 (1968), 620. Abstract \#657-24. [p. 1154]

1968b. An extension of Radon's theorem, Illinois J. Math. 12 (1968), 184-189. MR 37 \#824 [p. 1168]

E. Ja. Remez and A. S. SteY̌nberg,

1967. On a theorem on convex polyhedra in connection with the question of finding the totality of solutions of systems of linear inequalities, Ukrain. Mat. Ž. 19 (1967), no. 2, 74-89. (Russian) MR 35 \#3543. [p. 1134]

K. Reidemeister and K. Horneffer,

1968. Zur Farbung von Simplizialkomplexen der Sphare, Nachr. Akad. Wiss. Gottingen. Math.-Phys. Kl. II, 1968, 171-182. MR 38 \#5204. [p. 1183]

G. Ringel,

1956. Teilungen der Ebene durch Geraden oder topologische Geraden, Math. Z. 64 (1956), 79-102. MR 17, 651. [p. 1183]

1957. Über Geraden in allgemeiner Lage, Elem. Math. 12 (1957), 75-82. MR 19, 673. [p. 1183]

J. Riordan,

1966. The number of faces of simplicial polytopes, J. Combinatorial Theory 1 (1966), 82-95. MR 33 \#6501. [p. 1149]

R. R. Rottenberg,

1969. On finite sets of points in $P^{3}$, Technion Preprint Series, no. MT-31, Technion, Haifa, 1969. [p. 1171]

D. A. Rowland,

1968. An extension of Eberhard's theorem, M.Sci. Thesis, University of Washington, Seattle, Wash., 1968. [p. 1142]

H. Sachs,

1968. Ein von Kozyrev und Grinberg angegebener nicht-Hamiltonischer kubischer polanarer Graph, Beitrage zur Graphentheorie Internat. Kolloq. Manebach, 1967. Teubner, Leipzig, 1968, pp. 127-130. MR 39 \#5403. [p. 1144]

G. T. Sallee,

1966. A valuation property of Steiner points, Mathematika 13 (1966), 76-82. MR 33 \#7937. [p. 1166]

1967. Incidence graphs of convex polytopes, J. Combinatorial Theory 2 (1967), 466506. MR 35 \#7198. [p. 1134]

1968. Polytopes, valuations, and the Euler relation, Canad. J. Math. 20 (1968), 1412-1424. MR 38 \#605. [p. 1166]

1970. A non-continuous "Steiner point" (to appear). [p. 1163]

G. Sansone,

1928. Sui prismi e le piramidi regolari razionali, Period. Mat. (4) 8 (1928), 106116. [p. 1181]

L. A. Santal6,

1946. On the convex bodies of constant width in $E_{n}$, Portugal. Math. 5 (1946), 195201. MR 9, 526. [p. 1165]

P. Scherk,

1969. Über eine Klasse von Polyederfunktionalen, Comment. Math. Helv. 44 (1969), 191-201. [p. 1166]

V. Schlegel,

1891. Ueber die verschiedenen Formen von Gruppen, welche $r$ beliebige Punkte im 
n-dimensionalen Raum bilden konnen, Arch. Math. Phys. (2) 10 (1891), 283299. [p. 1173]

1893. Ueber Projectionen der mehrdimensionalen regelmässigen Körper, Jber. Deutsch. Math. Verein. 2 (1893), 66-69. [p. 1137]

K.-A. Schmitt,

1967. Hilbert spaces containing subspaces consisting of symmetry classes of convex bodies, Proc. Colloq. Convexity (Copenhagen, 1965) Kфbenhavns Univ. Mat. Inst., Copenhagen, 1967, pp. 278-280. [p. 1163]

1968. Kennzeichnung des Steinerpunktes konvexer Körper, Math. Z. 105 (1968),

R. Schneider, 387-392. MR 37 \#6834. [p. 1163]

1967a. Zu einem Problem von Shephard über die Projektionen konvexer Körper, Math. Z. 101 (1967), 71-82. MR 36 \#2059. [p. 1170]

1967b. Über die Durchschnitte translationsgleicher konvexer Körper und eine Klasse konvexer Polyeder, Abh. Math. Sem. Univ. Hamburg. 30 (1967), 118-128. MR 35 \#6029. [p. 1173]

1970. On Steiner points of convex bodies, Israel J. Math. (to appear). [p. 1163]

F. Schoblik,

1930. Zum Problem des Kartenfärbens, Jber. Deutsch. Math. Verein. 39 (1930), 51-52. [p. 1143]

P. H. Schoute,

1905. Mehrdimensionale Geometrie. Zweiter Teil: Die Polytope, Teubner, Leipzig, 1905. [p. 1137, p. 1173]

T. K. Sheng,

1966. Rational polygons, J. Austral. Math. Soc. 6 (1966), 452-459. MR 35 \#137. [p. 1181]

G. C. Shephard,

1964a. Approximation problems for convex polyhedra, Mathematika 11 (1964), 9-18. MR 30 \#2399. [p. 1163]

1966a. The Steiner point of a convex polytope, Canad. J. Math. 18 (1966), 1294-1300. MR 35 \#4814. [p. 1162, p. 1163]

1966b. A pre-Hilbert space consisting of classes of convex sets, Israel J. Math. 4 (1966), 1-10. MR 34 \#3435. [p. 1163]

1967a. An elementary proof of Gram's theorem for convex polytopes, Canad. J. Math. 19 (1967), 1214-1217. MR 37 \#822. [p. 1158]

1967b. Polytopes with centrally symmetric faces, Canad. J. Math. 19 (1967), 12061213. MR 36 \#4433. [p. 1170]

1968a. Angle deficiencies of convex polytopes, J. London Math. Soc. 43 (1968), 325336. MR 37 \#5784. [p. 1158, p. 1159, p. 1160, p. 1161]

1968b. A uniqueness theorem for the Steiner point of a convex region, J. London Math. Soc. 43 (1968), 439-444. MR 37 \#3447. [p. 1163]

1968c. Euler-type relations for convex polytopes, Proc. London Math. Soc. (3) 18 (1968), 597-606. MR 38 \#606. [p. 1164]

1968d. A theorem on cyclic polytopes, Israel J. Math. 6 (1968), 368-372. MR 39 \#6170. [p. 1150]

1968e. The mean width of a convex polytope, J. London Math. Soc. 43 (1968), 207209. MR 37 \#2087. [p. 1165]

1970a. Diagrams for positive bases (to appear). [p. 1171, p. 1176]

1970b. Spherical complexes and radial projections of polytopes, Israel J. Math. (to appear). [p. 1184] 
G. C. Shephard and R. J. Webster,

1965. Metrics for sets of convex bodies, Mathematika 12 (1965), 73-88. MR 32 \#2975. [p. 1163]

D. M. Y. Sommerville,

1927. The relations connecting the angle-sums and volume of a polytope in space of $n$ dimensions, Proc. Roy. Soc. London Ser. A 115 (1927), 103-119. [p. 1158]

S. K. Stein,

1951. Convex maps, Proc. Amer. Math. Soc. 2 (1951), 464-466. MR 12, 845. [p. 1138]

J. Steiner,

1830. Problème de situation, Ann. Math. Gergonne 19 (1830), 36; Cf: Ges. Werke. Vol. 1, 1881, p. 227. [p. 1173]

1840. Von dem Krümmungsschwerpunkte ebener Curven, J. Reine Angew. Math. 21 (1840), 33-63, 101-122; Cf: Ges. Werke. Vol. 2, Reiner, Berlin, 1882, pp. 99-159. [p. 1162]

E. Steinitz,

1906. Über die Eulersche Polyederrelationen, Arch. Math. Phys. (3) 11 (1906), 8688. [p. 1148]

1909. Über diejenigen konvexen Polyder mit $n$ Grenzflächen, welche nicht durch n-4 ebene Schnitte aus einen Tetraeder abgeleitet werden können, Arch. Math. Phys. (3) 14 (1909), 1-48. [p. 1171]

1913. Bedingt konvergente Reihen und konvexe Systeme, J. Reine Angew. Math. 143 (1913), 128-175; 144 (1914), 1-40; 146 (1916), 1-52. [p. 1171]

1922. Polyeder und Raumeinteilungen, Enzykl. Math. Wiss. 3 (1922), Geometrie part 3AB12, 1-139. [p. 1136, p. 1137]

E. Steinitz and H. Rademacher,

1934. Vorlesungen über die Theorie der Polyeder, Springer, Berlin, 1934. [p. 1137]

J. Stoer and C. Witzgall,

1970. Convexity and optimization in finite dimensions. I, Springer, New York and Berlin, 1970. [p. 1166]

M. Stojaković,

1959. Über die Konstruktion der ebenen Graphen, Univ. Beograd. Godišnjak Filozof. Fak. Novom Sadu 4 (1959), 375-378. MR 25 \#4518. [p. 1138]

F. Supnick,

1951 On the perspective deformation of polyhedra, Ann. of Math. (2) 53 (1951), 551-555. MR 12, 846. [p. 1184]

P. G. Tait,

1880. Remarks on the colouring of maps, Proc. Roy. Soc. Edinburgh 10 (1880), 501-503. [p. 1143]

W. T. Tutte,

1946. On Hamiltonian circuits, J. London Math. Soc. 21 (1946), 98-101. MR 8, 397. [p. 1143]

1956. A theorem on planar graphs, Trans. Amer. Math. Soc. 82 (1956), 99-116. MR 18, 408. [p. 1146]

1960a. Convex representations of graphs, Proc. London Math. Soc. (3) 10 (1960), 304-320. MR 22 \#5593. [p. 1138]

1960b. A non-Hamiltonian planar graph, Acta Math. Acad. Sci. Hungar. 11 (1960), 371-375. MR 25 \#4517. [p. 1143]

1961. A theory of 3-connected graphs, Nederl. Akad. Wetensch. Proc. Ser. A 64 = Indag. Math. 23 (1961), 441-455. MR 25 \#3517. [p. 1138] 
1963. How to draw a graph, Proc. London Math. Soc. (3) 13 (1963), 743-767. MR 28 \#1610. [p. 1138]

1968. On the enumeration of planar maps, Bull. Amer. Math. Soc. 74 (1968), 64-74. MR 36 \#1363. [p. 1173]

S. Ulam,

1960. A collection of mathematical problems, Interscience Tracts in Pure and Appl. Math., no. 8, Interscience, New York, 1960. MR 22 \#10884. [p. 1181]

M. Vaccaro,

1954. Sulla caratteristica dei complessi simpliciali $n$-dimensionali $\chi$-omogenei, Proc. Internat. Congress Math. (Amsterdam, 1954) vol. 2, pp. 261-262. [p. 1149]

1956. Sulla caratteristica dei complessi simpliciali x-omegenei, Ann. Mat. Pura Appl. (4) 41 (1956), 1-20. MR 17, 1120. [p. 1149]

W. Volland,

1957. Ein Fortsetzungssatz für additive Eipolyederfunktionale im euklidischen Raum, Arch. Math. 8 (1957), 144-149. MR 19, 1074. [p. 1166]

K. Wagner,

1936a. Bemerkungen zum Vierfarbenproblem, Jber. Deutsch. Math. Verein. 46 (1936), 26-32. [p. 1138]

1936b. Ein Satz iber Komplexe, Jber. Deutsch. Math. Verein. 46 (1936), 21-22. [p. 1143]

D. W. Walkup,

1970. The lower bound conjecture for 3- and 4-manifolds, Acta Math. (to appear). [p. 1153]

H. Walther,

1965. Ein kubischer, planarer, zyklisch fïnfach zusammenhängender Graph, der keinen Hamiltonkreis besitzt, Wiss. Z. Techn. Hochsch. Ilmenau 11 (1965), 163-166. MR 33 \#70. [p. 1144]

1966. Über das Problem der Existenz von Hamiltonkreisen in planaren regulären Graphen der Grade 3, 4, und 5, Thesis, Techn. Hochschule Ilmenau, 1966. [p. 1144]

1967. Über die Anzahl der Knotenpunkte eines längsten Kreises in planaren, kubischen, dreifach knotenzusammenhängenden Graphen, Studia. Sci. Math. Hungar. 2 (1967), 391-398. MR 36 \#1364. [p. 1146]

1968. On the problem of the existence of Hamilton-lines in planar regular graphs, Proc. Colloq. Theory of Graphs (Tihany, 1966) Academic Press, New York; Akad. Kiad6, Budapest, 1968, pp. 341-343. [p. 1144]

1969a. Über das Problem der Existenz von Hamiltonkreisen in planaren, regulären Graphen, Math. Nachr. 39 (1969), 277-296. [p. 1144]

1969b. Über die Nichtexistenz eines Knotenpunktes durch den alle längsten Wege eines Graphen gehen, J. Combinatorial Theory 6 (1969), 1-6. MR 38 \#4352. [p. 1147]

M. E. Watkins,

1968. On the existence of certain disjoint arcs in graphs, Duke Math. J. 35 (1968), 231-246. MR 36 \#6316. [p. 1134]

M. E. Watkins and D. M. Mesner,

1967. Cycles and connectivity in graphs, Canad. J. Math. 19 (1967), 1319-1328. MR 36 \#1355. [p. 1134]

H. Whitney,

1932. Non-separable and planar graphs, Trans. Amer. Math. Soc. 34 (1932), 339362. [p. 1136] 
L. Woo,

1969. An algorithm for straight-line representation of simple planar graphs, J. Franklin Inst. 287 (1969), 197-208. MR 39 \#4045. [p. 1138]

W.-T. Wu,

1965. A theory of imbedding, immersion, and isotopy of polytopes in a euclidean space, Science Press, Peking, 1965. MR 35 \#6146. [p. 1157]

J. Zaks,

1968. On minimal complexes and decompositions of $E^{n}, \mathrm{Ph} . \mathrm{D}$. Thesis, University of Washington, Seattle, Wash., 1968. [p. 1135]

1969. On a minimality property of complexes, Proc. Amer. Math. Soc. 20 (1969), 439-444. MR 39 \#946. [p. 1135]

1970a. The analogue of Eberhard's theorem for 4-valent graphs on the torus (to appear). [p. 1140]

1970b. On realizing symmetric 3-polytopes (to appear). [p. 1183]

1970c. The analogue of Eberhard's theorem for 4-valent graphs on the torus, Israel J. Math. (to appear). [p. 1183]

Michigan State University, East Lansing, Michigan 48823 